\title{
Astrocytes render memory flexible
}

\author{
Authors \\ Wuhyun Koh ${ }^{1,2,3}$, Mijeong Park ${ }^{1,4}$, Ye Eun Chun ${ }^{1,3}$, Jaekwang Lee ${ }^{3}$, Hyun Soo Shim ${ }^{4}$, Mingu \\ Gordon Park $^{2,5}$, Sunpil Kim ${ }^{2,3,5}$, Hyunji Kang ${ }^{1,2}$, Soo-Jin Oh ${ }^{4,6}$, Junsung Woo ${ }^{1,3}$, Heejung Chun ${ }^{2,3}$, \\ Seungeun $\mathrm{Lee}^{7}$, Jinpyo $\mathrm{Hong}^{3}$, Jiesi Feng ${ }^{8}$, Yulong $\mathrm{Li}^{8}$, Hoon $\mathrm{Ryu}^{4}$, Jeiwon $\mathrm{Cho}^{9}$, and C. Justin \\ $\operatorname{Lee}^{1,2,10, *}$
}

\section{Affiliation}

${ }^{1}$ Department of Neuroscience, Division of Bio-Medical Science \& Technology, KIST School, Korea University of Science and Technology, Seoul 02792, South Korea

${ }^{2}$ Center for Cognition and Sociality, Institute for Basic Science (IBS), Daejeon 34126, South Korea ${ }^{3}$ Center for Functional Connectomics, Brain Science Institute, Korea Institute of Science and Technology (KIST), Seoul 02792, South Korea

${ }^{4}$ Center for Neuroscience, Brain Science Institute, Korea Institute of Science and Technology (KIST), Seoul 02792, South Korea

${ }^{5}$ KU-KIST Graduate School of Converging Science and Technology, Korea University, Seoul 02841, South Korea

${ }^{6}$ Convergence Research Center for Diagnosis, Treatment and Care System of Dementia, Korea Institute of Science and Technology (KIST), Seoul 02792, South Korea

${ }^{7}$ Virus Facility, Research Animal Resource Center, Korea Institute of Science and Technology (KIST), Seoul 02792, South Korea

${ }^{8}$ State Key Laboratory of Membrane Biology, Peking University School of Life Sciences, Beijing 100871, China

${ }^{9}$ Brain and Cognitive Science, Scranton College, Ewha Womans University, Seoul 03760, South Korea

${ }^{10}$ Lead Contact

${ }^{*}$ Correspondence: cjl@ibs.re.kr (C.J.L.)

\section{Highlights}

- Astrocytes regulate NMDAR tone via Best1-mediated glutamate and D-serine release

- Activation of astrocytic a1-AR induces heterosynaptic LTD via NMDAR tone

- Heterosynaptic LTD is required for repotentiation LTP and spatial reversal learning

- Astrocytic regulation of NMDAR tone is critical for metaplasticity and flexible memory 


\section{Summary}

2 Cognitive flexibility is an essential ability to adapt to changing environment and circumstances. NMDAR

3 has long been implicated in cognitive flexibility, but the precise molecular and cellular mechanism is not

4 well understood. Here, we report that astrocytes regulate NMDAR tone through Best1-mediated

5 glutamate and D-serine release, which is critical for cognitive flexibility. Co-release of D-serine and

6 glutamate is required for not only homosynaptic LTD but also heterosynaptic LTD, which is induced at

7 unstimulated synapses upon release of norepinephrine and activation of astrocytic $\alpha 1-$ AR during

8 homosynaptic LTP. Remarkably, heterosynaptic LTD at unstimulated synapses during memory

9 acquisition is required for later repotentiation LTP during reversal learning, laying a foundation for flexible

10 memory and cognitive flexibility. Our study sheds light on the pivotal role of astrocytes in orchestrating

11 multiple synapses during memory formation and determining the fate of consolidated memory to be

12 retained as a flexible memory.

14 Keywords: Cognitive flexibility, Astrocyte, Best1, NMDAR tone, D-serine, Glutamate, Hippocampus,

15 LTD, Metaplasticity, Norepinephrine 


\section{Introduction}

17 The flexibility of memory is as important as the formation of memory because an environment and circumstances are not static, but dynamically changing. When necessary, previously acquired memories should be flexibly adjusted to adapt to the changing environment. This ability is generally termed as cognitive flexibility (Tello-Ramos et al., 2019). Cognitive flexibility has been reported to decline in several

21 diseases, for instance, autism spectrum disorder (ASD) (D'Cruz et al., 2013), schizophrenia (Wobrock et

22 al., 2009), and early stages of Alzheimer's disease (AD) (Etienne et al., 2013; Guarino et al., 2018), in

24 Huang et al., 2012; Lee and Zhou, 2019). However, little is known about how NMDAR hypofunction

25 affects cognitive flexibility. In the hippocampus, NMDAR-dependent long-term depression (LTD) is

26 proposed to be associated with spatial reversal learning (Duffy et al., 2008; Li et al., 2015; Morice et al.,

27 2007; Nicholls et al., 2008), a hippocampus-dependent form of cognitive flexibility (Izquierdo et al., 2017).

28 Of the synaptic NMDAR (synNMDAR) and extrasynaptic NMDAR (exNMDAR) classified according to

29 location, the latter has been suggested to be particularly important for LTD in the hippocampus (Liu et al.,

30 2013; Lu et al., 2001). The current mediated by exNMDAR is also referred to as "tonic NMDAR" or

31 "NMDAR tone" due to its slow-time-scale or continuous occupancy/activation of exNMDAR by an ambient

32 glutamate (Le Meur et al., 2007) and co-agonists (i.e. D-serine and glycine). Although exNMDAR appears

33 to be crucial for LTD and cognitive flexibility, it has not been yet clear which cell regulates NMDAR tone

34 for exNMDAR currents in the brain.

35 D-serine, one of the co-agonists that could constitute NMDAR tone, has been extensively investigated 36 in hippocampal LTD and spatial reversal learning. For example, exogenous D-serine was shown to 37 augment hippocampal LTD (Duffy et al., 2008; Zhang et al., 2008) and spatial reversal learning (Duffy et 
al., 2008). In addition, a transgenic mouse with a loss-of-function mutation in D-amino acid oxidase

(DAAO), a key catabolic enzyme for D-serine, showed enhanced spatial reversal learning (Labrie et al., 2009). In contrast, a depletion of endogenous D-serine by DAAO was shown to impair and reduce hippocampal LTD (Papouin et al., 2012; Zhang et al., 2008). While these results suggest that D-serinemediated NMDAR tone can facilitate spatial reversal learning, the precise molecular and cellular mechanism of how endogenous D-serine is regulated and facilitates spatial reversal learning is not well understood. Importantly, it has been still unclear and controversial whether the cellular source of D-serine is astrocytes (Papouin et al., 2017) or neurons (Wolosker et al., 2017). an increase in hippocampal NMDAR tone (Shen et al., 2017) and hippocampal LTD (Navarrete et al., 2019). This increase in NMDAR tone was reduced by the treatment of NPPB (Shen et al., 2017), which blocks anion channels including the $\mathrm{Ca}^{2+}$-activated, glutamate-permeable anion channel Best1 (Oh and Lee, 2017), suggesting that glutamate released through Best1 from astrocytes (Park et al., 2013; Woo et al., 2012) contributes to hippocampal NMDAR tone and LTD induction. However, in place of exogenous

52 ChR2, which induces hippocampal LTD, an endogenous molecule that activates astrocytes has not been 53 identified yet. Previously, it has been reported that chemogenetic activation of locus coeruleus (LC) can

54 restore spatial reversal learning in early stages of AD model (Rorabaugh et al., 2017). Interestingly,

55 norepinephrine (NE), synthesized in LC neurons, is known to induce hippocampal LTD through a1-

56 adrenergic receptor ( $\alpha 1-A R$ ) (Dyer-Reaves et al., 2019; Scheiderer et al., 2004), which is predominantly

57 localized in astrocytes (Hertz et al., 2010). It has been later demonstrated that astrocytes respond to NE

58 through a1-AR by an increase in cytosolic $\mathrm{Ca}^{2+}$ (Ding et al., 2013), which can possibly open Best1 in 
$\mathrm{Ca}^{2+-}$-dependent manner. However, it is not known whether NE, through activation of astrocytic $\alpha 1-\mathrm{AR}$, can cause an increase in NMDAR tone and hippocampal LTD via Best1.

Activation of astrocytes was shown to induce not only homosynaptic LTD (Navarrete et al., 2019), but also heterosynaptic LTD (Chen et al., 2013a), which was initially documented to occur at the unstimulated synapses accompanying homosynaptic long-term potentiation (LTP) at the stimulated synapses (Lynch et al., 1977; Scanziani et al., 1996). In early times, homosynaptic LTD induced by low-frequency stimulation (LFS) has been thought to be associated with spatial reversal learning (Nicholls et al., 2008). In contrast, heterosynaptic LTD has been suggested to enable metaplasticity (Chen et al., 2013b), which is defined as a plasticity of synaptic plasticity (Abraham and Bear, 1996; Hulme et al., 2014). However, it is not

68 known whether heterosynaptic LTD is associated with spatial reversal learning. Paradoxically, it has been also demonstrated that spatial reversal learning is either enhanced in mice with decreased homosynaptic LTD (Zhang and Wang, 2013) or diminished in mice with increased homosynaptic LTD (Rutten et al.,

71 2011). These findings challenge the initial notion that homosynaptic LTD is responsible for spatial

72 reversal learning and raise the possibility of heterosynaptic LTD to be more closely linked to spatial

73 reversal learning. Nevertheless, it has not been studied whether heterosynaptic LTD through activation of 74 astrocytes is associated with spatial reversal learning.

75 In the present study, we have investigated how astrocytes regulate heterosynaptic LTD and metaplasticity, and thereby contribute to spatial reversal learning. We employed cell-type specific genetic

77 manipulations, ex vivo electrophysiological recordings, sniffer patch recordings, cutting-edge biosensor

78 for NE, and behavioral assays to investigate whether astrocytes can regulate NMDAR tone by releasing

79 D-Serine and glutamate. Subsequently, we further investigated the role for NMDAR tone in the 
bioRxiv preprint doi: https://doi.org/10.1101/2021.03.25.436945; this version posted March 25, 2021. The copyright holder for this preprint (which was not certified by peer review) is the author/funder. All rights reserved. No reuse allowed without permission.

81 indeed dynamically control heterosynaptic LTD during an induction of homosynaptic LTP through NMDAR

82 tone regulation via Best1. Furthermore, we found that this heterosynaptic LTD becomes a basis for the

83 flexible memory which is required for spatial reversal learning and cognitive flexibility. 
84

\section{Results}

\section{Astrocytes regulate hippocampal NMDAR tone through Best1}

It has been suggested that astrocytic $\mathrm{Ca}^{2+}$ is an important signaling molecule for the release of gliotransmitters (Sahlender et al., 2014; Semyanov et al., 2020), including glutamate (Lee et al., 2007; Parpura and Haydon, 2000) and D-serine (Henneberger et al., 2010; Takata et al., 2011). Thus, we hypothesized that if hippocampal astrocytes regulate NMDAR tone in a $\mathrm{Ca}^{2+}$-dependent manner, an inhibition of astrocytic $\mathrm{Ca}^{2+}$ would lead to reduced NMDAR tone. To investigate whether astrocytes regulate NMDAR tone in hippocampus, exNMDAR current was measured in CA1 pyramidal neuron, while $\mathrm{Ca}^{2+}$ signaling in nearby astrocytes is suppressed by $\mathrm{Ca}^{2+}$-clamping. To measure exNMDAR current, a shift in the whole-cell current in response to a treatment of $50 \mu \mathrm{M}(2 \mathrm{R})$-amino-5-phosphonovaleric acid (APV), the NMDAR blocker, was quantified under voltage-clamping at $+40 \mathrm{mV}$ in the presence of other inhibitors $(20 \mu \mathrm{M}$ CNQX for AMPAR, $10 \mu \mathrm{M}$ Bicuculline for GABA $\mathrm{R}, 10 \mu \mathrm{M}$ CGP 55845 for GABA $R, 10$ $\mu \mathrm{M}$ Strychnine for GlyR) (Figure 1A). To inhibit astrocytic $\mathrm{Ca}^{2+}$ signaling, BAPTA, a $\mathrm{Ca}^{2+}$ chelator, and Alexa Fluor 488, a fluorescent indicator, were loaded into astrocytes through a patch pipette (Figures 1B and S1A), as previously described (Kwak et al., 2020; Serrano et al., 2006; Shigetomi et al., 2008). We observed that exNMDAR current was significantly reduced when astrocytes were loaded with BAPTA (+BAPTA), compared to without BAPTA (-BAPTA) condition (Figures 1C and 1D). These results indicate that astrocytes regulate hippocampal NMDAR tone in a $\mathrm{Ca}^{2+}$-dependent manner.

It has been previously reported that Bafilomycin A treatment did not affect exNMDAR current, indicating that vesicular release does not majorly contribute to exNMDAR current (Le Meur et al., 2007). Based on this finding, we hypothesized that a non-vesicular, $\mathrm{Ca}^{2+}$-dependent channel-mediated mechanism might be involved, and tested the possibility that Best1-mediated gliotransmission contributes 
to exNMDAR current using Best1 knockout (KO) mice (Marmorstein et al., 2006). We found that exNMDAR current was significantly decreased in Best1 KO, compared to the wild type (WT) mice (Figures 1E-1F). This decrease in exNMDAR current in Best1 KO was on average $63.9 \%$ of that in WT (Figure 1G). To test if the decreased exNMDAR current in Best1 $\mathrm{KO}$ is due to the lack of Best1-mediated gliotransmission in astrocytes, we over-expressed Best1 full-clone under GFAP promoter in hippocampal astrocytes of Best1 KO by injecting AAV-GFAP-Best1-IRES-EGFP virus into CA1 hippocampus (Figures

$1121 \mathrm{H}, 1 \mathrm{I}$ and S1B). We found that the decreased exNMDAR current in Best1 KO was significantly recovered

113 in astrocyte-specific Best1 rescue group (GFAP-Best1), but not in control group (GFAP-GFP) (Figures 1J

114 and 1K), indicating that the decreased exNMDAR current in Best1 KO is caused by reduced Best1-

115 mediated gliotransmission from astrocytes. The decreased exNMDAR current was not due to a

116 decreased protein expression of NMDAR, as evidenced by consistent protein expression level of GluN1,

117 an indispensable subunit for functional NMDAR (Figure S2). These results suggest that astrocytic $\mathrm{Ca}^{2+-}$

118 activated anion channel Best1 mediates NMDAR tone in hippocampus.

119 NMDAR tone can be attributed to both glutamate and NMDAR co-agonist (i.e. D-serine or glycine), as 120 both are required for NMDAR activation. To dissect the contribution of glutamate component to NMDAR

121 tone, exNMDAR glycine modulatory site (GMS) was fully occupied by an exogenous application of $100 \mu \mathrm{M}$

122 D-serine (Figure 1L). The application of D-serine increased exNMDAR current in WT by $47.9 \%$ (before,

$12315.9 \pm 1.4 \mathrm{pA}$; after, $23.5 \pm 1.9 \mathrm{pA}$; mean \pm SEM) and Best1 KO by $105 \%$ (before, $7.2 \pm 1.8 \mathrm{pA}$; after,

$124 \quad 14.7 \pm 2.4 \mathrm{pA}$; mean \pm SEM) (Figures $1 \mathrm{~L}$ and $1 \mathrm{M}$ ), implying that GMS was not fully occupied in both

125 genotypes under basal condition. More importantly, we found that the GMS-saturated exNMDAR current

126 was significantly reduced in Best1 $\mathrm{KO}$ to $62.6 \%$ (Figure $1 \mathrm{M}$ ), indicating a reduced ambient level of

127 glutamate in Best1 KO. Remarkably, we observed that when D-serine was applied to Best1 KO, the 
128 GMS-saturated exNMDAR current was significantly recovered to the level of WT (Figure 1M), indicating

129 that D-serine supplement is sufficient to fully rescue the impaired NMDAR tone of Best1 KO to the WT

130 level. To measure the ambient level of NMDAR co-agonist, GMS-occupancy of exNMDAR was calculated

131 by dividing the basal exNMDAR current by GMS-saturated exNMDAR current for each genotype (Figures

$1321 \mathrm{M}$ and $1 \mathrm{~N})$. The percentage of GMS-occupancy of exNMDAR was significantly reduced in Best1 KO by

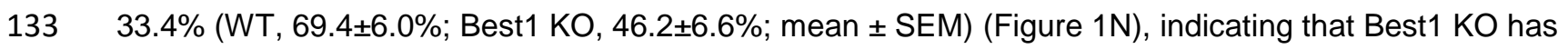

134 reduced NMDAR co-agonist as well as glutamate. Furthermore, we observed a similarly reduced GMS-

135 occupancy of synNMDAR in Best1 KO as well as in Best1 gene-silencing condition, which was fully

136 rescued by an astrocyte-specific Best1 rescue (Figures S3A-S3N). Taken together, these results indicate

137 that astrocytes regulate NMDAR tone through Best1 by modulating the ambient level of both glutamate

138 and co-agonist. 
A

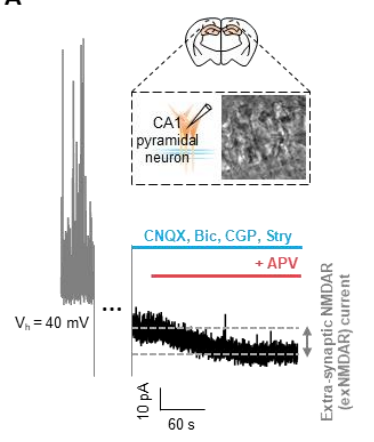

E

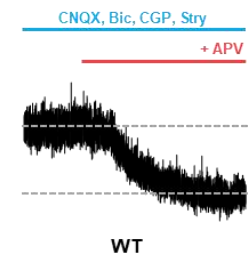

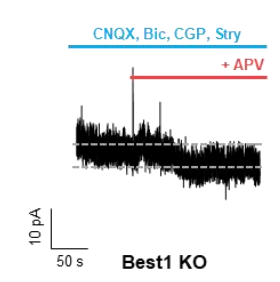

H

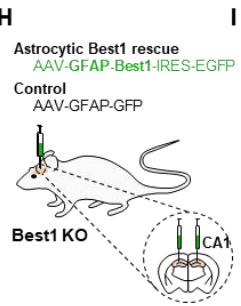

L

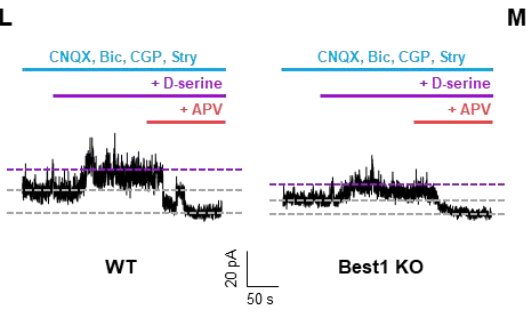

M

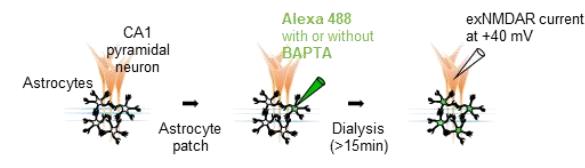

C
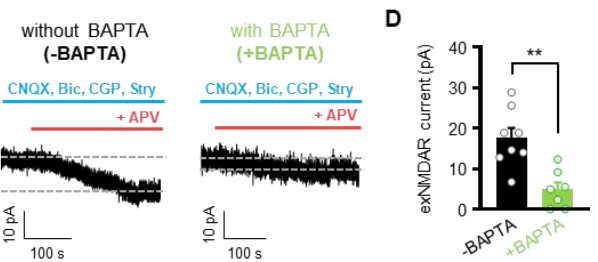

$\mathbf{F}$
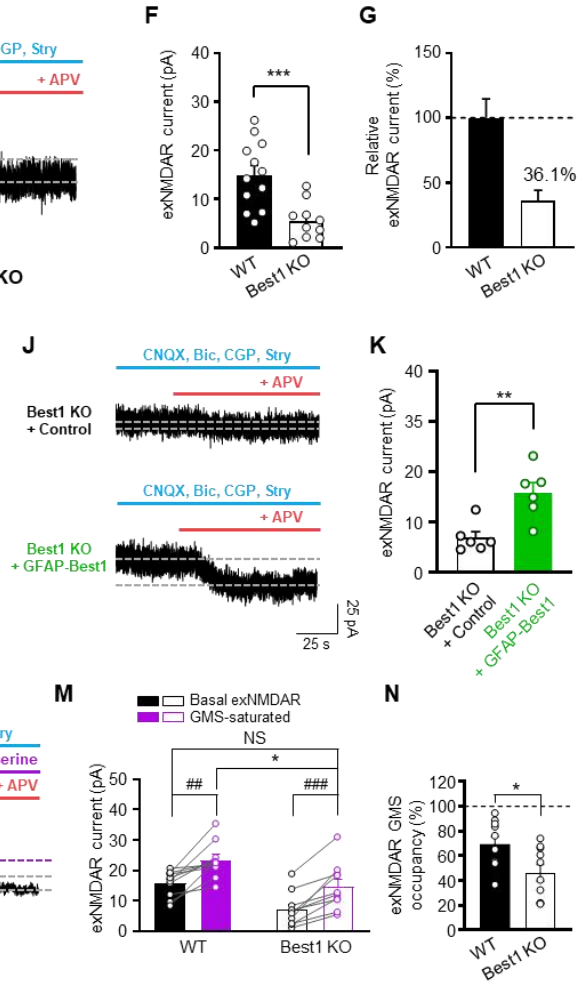

K

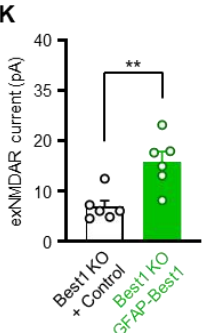

N

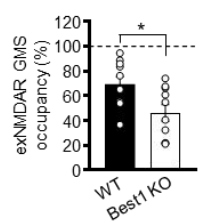

Figure 1. Astrocytes regulate hippocampal NMDAR tone through Best1.

(A) Extrasynaptic NMDAR (exNMDAR) current recording in hippocampal CA1 pyramidal neuron. To isolate NMDARmediated current, CNQX, Bicuculline (Bic.), CGP55845 (CGP.), and Strychnine (Stry.) were used. APVsensitive current in voltage holding at $+40 \mathrm{mV}$. (B) Astrocytic $\mathrm{Ca}^{2+}$ chelation with BAPTA dialysis. (C)

Representative traces of exNMDAR current with or without BAPTA dialysis. (D) Summary graph of exNMDAR current with or without BAPTA dialysis. (E-G) Representative traces of exNMDAR current $(E)$, summary graph of exNMDAR current $(F)$, and relative exNMDAR current to WT $(G)$ in WT and Best1 KO. (H-K) Scheme of astrocytic Best1 rescue in CA1 of Best1 $\mathrm{KO}(\mathrm{H})$, images showing virus expression (I), representative traces of exNMDAR current $(\mathrm{J})$ and summary graph of exNMDAR current in each condition (K). (L) Application of $100 \mu \mathrm{M}$ D-serine during exNMDAR measurement. (M) Summary graph of exNMDAR current before (black) and after D-serine treatment (purple) in each condition. (N) Estimated exNMDAR GMS occupancy

(\%). Individual dots refer to cells. Data are represented as mean \pm SEM. ${ }^{*} \mathrm{p}<0.05 ;{ }^{* *} \mathrm{p}<0.01 ;{ }^{* * *} \mathrm{p}<0.001$; Mann Whitney test (D and $\mathrm{K}$ ) or unpaired-t test $(\mathrm{F}, \mathrm{M}, \mathrm{N})$. \#p $<0.05$; \#\# $<0.01$; \#\#p $<0.001$; paired t-test $(\mathrm{M})$. 


\section{D-serine and glutamate are co-released from astrocyte through Best1}

140 To investigate which co-agonist, D-serine or glycine, is released from astrocytes in a Ca²+-dependent

141 manner, we conducted a sniffer-patch experiment with a solitary primary cultured astrocyte and a sensor

142 cell, as previously described (Lee et al., 2007; Woo et al., 2012). To induce $\mathrm{Ca}^{2+-d e p e n d e n t ~ r e l e a s e ~ f r o m ~}$

143 an astrocyte, TFLLR, an agonist of protease-activated receptor-1 (PAR-1), was locally applied, and

144 sensor current elicited by a release of glycine or D-serine was recorded from a HEK293T cell expressing

145 a biosensor (Figure 2A). To discriminate between glycine and D-serine, we utilized either NMDAR (NR1-

146 1a and chimeric NR2A(2D-S1)) (Chen et al., 2008) for the detection of both glutamate and co-agonist, or

147 glycine receptor (human glycine receptor alpha 1; hGlyR a1 L261F) (Laube et al., 2000) for the detection

148 of glycine, but not D-serine, as the biosensor (Figure 2B). When astrocytic $\mathrm{Ca}^{2+}$ was elicited by TFLLR,

149 we observed a significant NMDAR-sensor-current, whereas glycine-receptor-sensor-current was

150 minimally observed (Figures $2 \mathrm{C}$ and $2 \mathrm{D}$ ), indicating that an astrocyte releases glutamate together with an

151 NMDAR co-agonist other than glycine, most likely D-serine. To test whether this NMDAR co-agonist is

152 indeed D-serine, we expressed shRNA for serine racemase (SR), D-serine synthesizing enzyme that

153 converts L-serine to D-serine, to gene-silence SR (Figures 2E, S4A-S4E). SR shRNA-expressing

154 astrocyte showed a significantly decreased NMDAR-sensor-current compared to control shRNA-

155 expressing astrocyte (Figure 2E). This decreased NMDAR current was recovered by a 5-minute

156 incubation with $100 \mu \mathrm{M}$ D-serine on the same cell (Figure 2F). These results demonstrate that an

157 astrocyte releases $\mathrm{D}$-serine, not glycine, in a $\mathrm{Ca}^{2+}$-dependent manner to activate NMDAR.

158 We have previously reported that the astrocytic $\mathrm{Ca}^{2+}$-activated anion channel Best 1 is permeable to

159 glutamate and mediates the release of glutamate from astrocytes to target synNMDAR (Park et al., 2015;

160 Woo et al., 2012). Thus, we examined a possibility that D-serine could directly permeate Best1. To 
161 estimate the relative permeability of D-serine to Best1, we recorded $\mathrm{Ca}^{2+}$-activated Best1-mediated

162 current with serial substitutions of chloride in internal pipette solution with equivalent concentrations of D-

163 serine (Figures $2 \mathrm{G}$ and $2 \mathrm{H}$ ), as previously described for glutamate (Park et al., 2009). The gray dotted

164 lines indicate the predicted reversal potential, $E_{\text {rev }}$ by the Goldman-Hodgkin- Katz equation when D-serine

165 is as permeable as $\mathrm{Cl}^{-}\left(\mathrm{P}_{\mathrm{D} \text {-serine }} / \mathrm{P}_{\mathrm{Cl}}=1\right)$ and when $\mathrm{D}$-serine is not permeable at all $\left(\mathrm{P}_{\mathrm{D} \text {-serine }} / \mathrm{p}_{\mathrm{Cl}}=0\right)($ Figure

$1662 \mathrm{H})$. We found that the observed Erev's fell somewhere in between the two dotted lines, indicating that D-

167 serine permeability is greater than zero but less than that of chloride (Figure $2 \mathrm{H}$ ). At the intracellular D-

168 serine concentration of $90 \mathrm{mM}$, the permeability ratio of $\mathrm{D}$-serine to chloride $\left(\mathrm{P}_{\mathrm{D}-\text { serine }} / \mathrm{P}_{\mathrm{Cl}}\right)$ was estimated

169 as 0.69 , according to the Goldman-Hodgkin-Kats equation (Figure $2 \mathrm{H}$ ). These results indicate that Best1

170 has a substantial permeability to D-serine.

171 Considering the fact that Best1 is permeable to both D-serine and glutamate, we next asked whether

172 D-serine and glutamate can be co-released through Best1. To directly test this possibility, we employed

173 two-cell-sniffer-patch technique, consisting of a source cell (HEK293T cell) expressing the full-clone of

174 mouse Best1 (mBest1) and a sensor cell (HEK293T cell) expressing NR1/NR2A(2D-S1) (Figure 2I). Pre-

175 rupture configuration for the source cell was prepared by forming a gigaseal with an internal pipette

176 solution containing $70 \mathrm{mM}$ glutamate with or without $70 \mathrm{mM} \mathrm{D}$-serine, and corresponding sensor current

177 was measured during a membrane-rupture of the source cell. For an immediate activation of mBest1 in

178 the source cell upon membrane-rupture, the internal pipette solution contained $4.5 \mu \mathrm{M}$ free $\mathrm{Ca}^{2+}$. We

179 found that upon the membrane rupture of the source cell expressing WT mBest1, a significant sensor

180 NMDAR-current was observed only when the source-cell internal pipette solution contained both

181 glutamate and D-serine (Figure 2J and 2K), indicating that Best1 releases both glutamate and D-serine

182 simultaneously. This Best1-mediated co-release of glutamate and D-serine was absent when the source 

cell expressed mBest1-W93C, a pore-mutant form of mBest1 (Figure $2 \mathrm{~J}$ and $2 \mathrm{~K}$ ), further strengthening

184 the concept of a co-release of glutamate and D-serine through Best1.

185 Finally, we tested the concept of co-release of glutamate and D-serine through Best1 in astrocytes. To

186 test the idea, sniffer-patch experiment was performed with Best1 shRNA-expressing cultured solitary

187 astrocytes (Figures 2L). Best1 shRNA-expressing astrocytes showed almost complete elimination of the

188 sensor-NMDAR-current, which was fully reconstituted by a co-expression of shRNA-insensitive form of

189 mBest1, whereas co-expression of shRNA-insensitive mBest1-W93C showed no recovery (Figure 2M

190 and 2N). Taken together, these results suggest that astrocytes co-release D-serine and glutamate

191 through Best1 in a $\mathrm{Ca}^{2+}$-dependent manner to activate adjacent NMDAR and mediate NMDAR tone in the

192 hippocampus. 
A
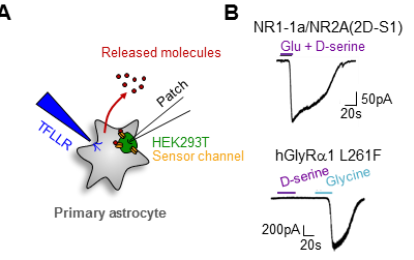

C
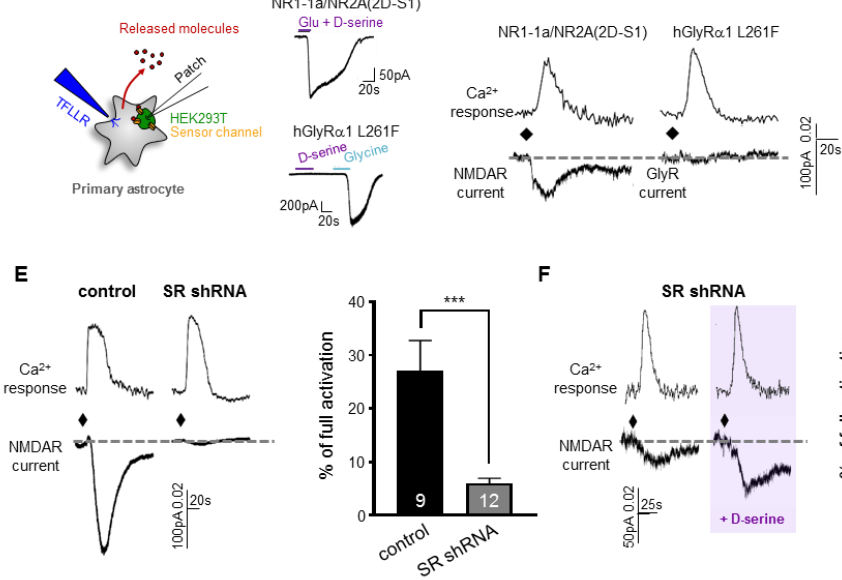

$\mathbf{F}$

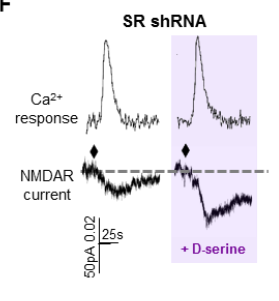

G
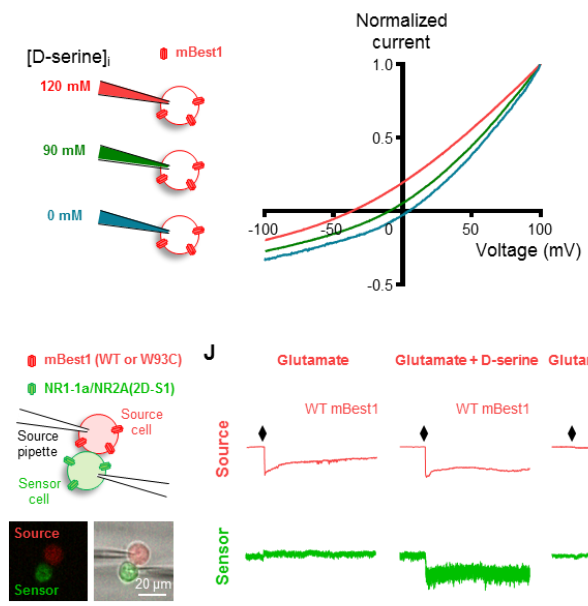

L

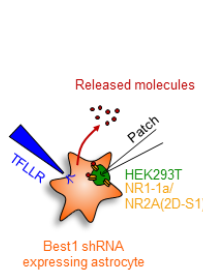

M

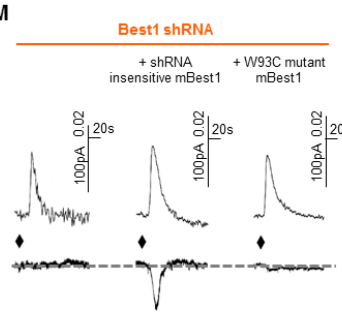

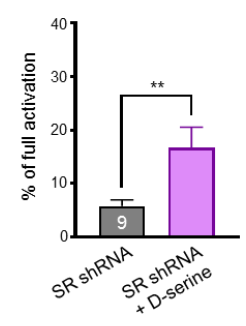

H
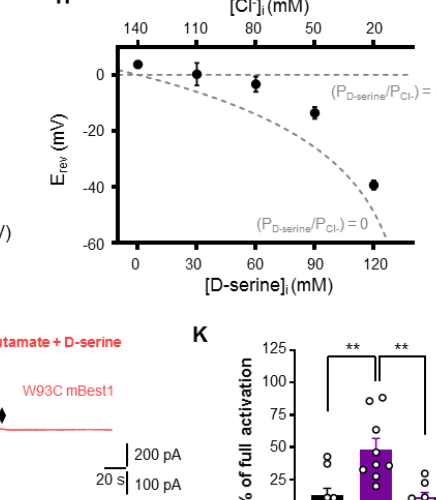

K

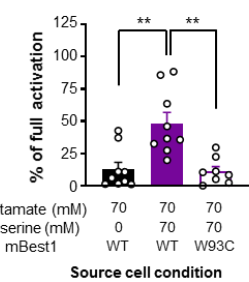

\section{西}
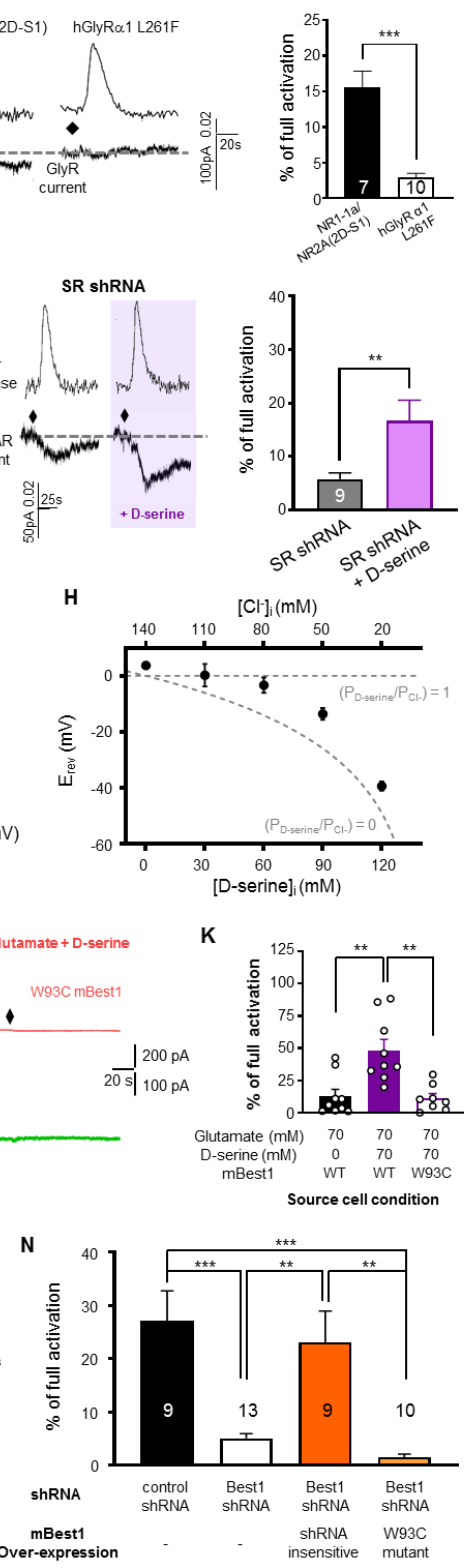

Figure 2. Astrocyte co-releases Dserine and glutamate through Best1 to activate NMDAR.

(A) Scheme of sniffer patch using primary astrocyte and sensor cell. (B) Validation of sensor channels. Upper: NR1-1a/NR2A(2D-S1)-mediated responsive current to glutamate and $D$ serine. Lower: hGlyRa1 L261Fmediated responsive current to $D$ serine and glycine, respectively. (C) Representative traces of $\mathrm{Ca}^{2+}$ response in astrocyte, and responsive sensor current from sensor cell expressing NR1-1a/NR2A(2D-S1) or hGlyRa1 L261F. (D) Summary graph of the peak amplitude normalized to full activation in sensor cell with NR1-1a/NR2A(2DS1) or hGlyRa1 L261F. (E) Left, representative traces of SR knockdown and control. Right, summary graph of the normalized peak amplitude in each condition. (F) Left, representative traces of SR knockdown before and after 100 $\mu \mathrm{M}$ D-serine treatment. Right, summary graph of the normalized peak amplitude in each condition. (G) I-V relationship in HEK293T cell expressing mouse Best1 (mBest1) in the presence of $\mathrm{Ca}^{2+}(\sim 4.5$ $\mu \mathrm{M})$ and varying intracellular concentration of D-serine. $(H)$ Dependence of current reversal potential $\left(E_{\text {rev }}, m V\right)$ on intracellular $D$ serine concentration. Gray dotted lines:

predicted $E_{\text {rev }}$ by the Goldman-Hodgkin- Katz equation when D-serine is as permeable as $\mathrm{Cl}^{-}\left(p_{\mathrm{D} \text {-serine }} / \mathrm{p}_{\mathrm{Cl}}=1\right)$ and when $\mathrm{D}$-serine is not permeable at all $\left(\mathrm{p}_{\mathrm{D}-\text { serine }} / \mathrm{p}_{\mathrm{Cl}}=0\right)$. (I) Scheme of two cell assay. Source cell expressing mBest1 WT or W93C mutant. (J) Representative traces of currents simultaneously recorded from source (red) and sensor (green) cells. (K) Summary graph of the normalized peak amplitude in each condition. (L) Sniffer patch with Best1 shRNA expressing astrocyte. (M) Representative traces of Best1 knockdown without or with either over-expression of shRNA insensitive form or W93C mutant form of Best1. (N) Summary graph of the normalized peak amplitude. Individual dots and numbers refer to cells. Data are represented as mean \pm SEM. ${ }^{*} p<$ $0.05 ;{ }^{* *} \mathrm{p}<0.01 ;{ }^{* * *} \mathrm{p}<0.001$; unpaired-t test ( $\mathrm{D}$ and $\left.\mathrm{E}\right)$, paired t-test $(\mathrm{F})$, and one-way ANOVA with Tukey's multiple comparison test $(\mathrm{K}$ and $\mathrm{N})$. 


\section{Decreased NMDAR tone leads to impaired LTD in hippocampus}

194 Equipped with the molecular and genetic tools to regulate NMDAR tone via Best1, we examined the

195 potential role of NMDAR tone in synaptic plasticity. To assess synaptic plasticity, we performed field

EPSP (fEPSP) recordings of the Schaffer collateral pathway at CA3-CA1 synapses, as previously

197 described (Nam et al., 2019; Park et al., 2015). We found that low frequency stimulation (LFS, 900 stimuli

198 at $1 \mathrm{~Hz}$ )-induced LTD was completely impaired in Best1 KO (Figures 3A-3C, S5C and S5D), whereas

199 effects produced by high frequency stimulation (HFS, 100 stimuli at $100 \mathrm{~Hz}$ ) or $10 \mathrm{~Hz}$ stimulation $(900$

200 stimuli at $10 \mathrm{~Hz}$ ) showed no difference between WT and Best1 KO (Figures 3C, S5A and S5B). These

201 results suggest that regulation of NMDAR tone through Best1 is critical for the induction of LTD, but not

202 LTP in the hippocampus. To test if the impaired LTD in Best1 KO is due to the lack of Best1-mediated co-

203 release of D-serine and glutamate in astrocytes, we over-expressed Best1 in hippocampal astrocytes by

204 injection of AAV-GFAP-Best1-IRES-EGFP virus into hippocampal CA1 of Best1 KO (Figures 1H-1K, S1D

205 and S1E) and examined LFS-induced LTD (Figures 3D-3F). We found that hippocampal LTD in Best1 KO

206 was fully restored by astrocytic Best1 rescue (GFAP-Best1), but not by control virus (GFAP-GFP)

207 (Figures 3E and 3F). These results indicate that astrocytic Best1 is sufficient for hippocampal LTD,

208 possibly via regulation of NMDAR tone.

209 It has been previously reported that depletion of D-serine impairs (Zhang et al., 2008) or reduces LTD

210 (Papouin et al., 2012). However the cellular source of D-serine was not determined. Potential sources of

211 D-serine includes astrocyte (Papouin et al., 2017) and neuron (Wolosker et al., 2017). To examine the

212 cellular source of D-serine required for LTD induction, we inhibited D-serine synthesis in a cell-type

213 specific manner with a Cre recombinase (Cre)-dependent SR shRNA expressing (AAV-pSico-RED SR

214 shRNA) virus, in combination with either astrocyte-specific (AAV-GFAP-Cre) or excitatory neuron-specific 
215 (AAV-CaMKIla-Cre) virus (Figure 3G). We found that astrocytic SR knockdown (aSR KD) eliminated LTD,

216 whereas neuronal SR knockdown (nSR KD) did not (Figures 3H and 3I). These results indicate that

217 astrocytic D-serine, but not neuronal D-serine, is critical for LTD induction. Finally, we tested whether the

218 impaired LTD in Best1 KO can be restored by increasing NMDAR tone during LTD induction. To increase

219 NMDAR tone in Best1 KO, $20 \mu \mathrm{M}$ D-serine was applied during LFS (Figure 3J). We found that application

220 of D-serine significantly restored the impaired LTD in Best1 KO (Figures 3J and 3K), indicating that

221 NMDAR tone during LFS is critical for hippocampal LTD. Taken together, these results indicate that

222 NMDAR tone mediated by astrocytic D-serine during LFS causes hippocampal LTD. 
A

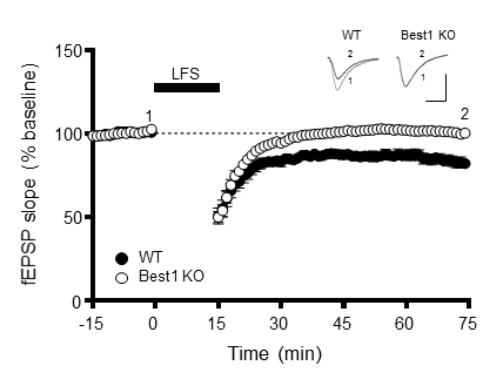

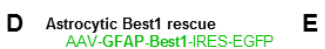
AAV-GFAP-Best1-
Control
AAV-GFAP-GFP

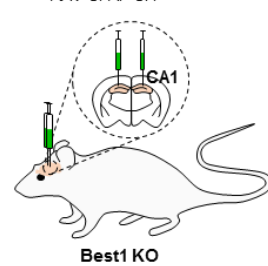

Best1 Ko

G $\begin{gathered}\text { Expression of Serine } \\ \text { Racemase (SR) shRNA }\end{gathered}$

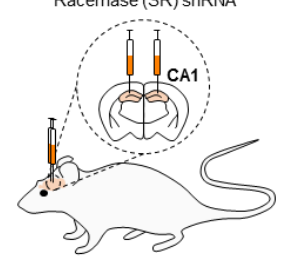

WT

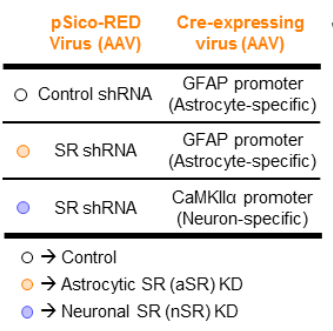

B

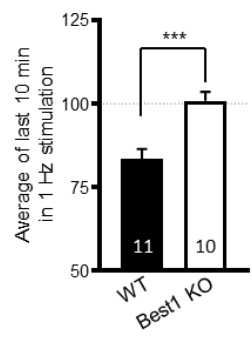

C

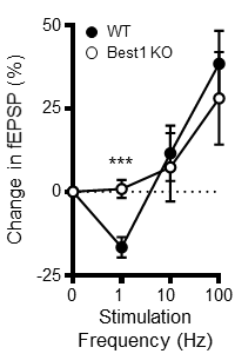

$\mathbf{F}$
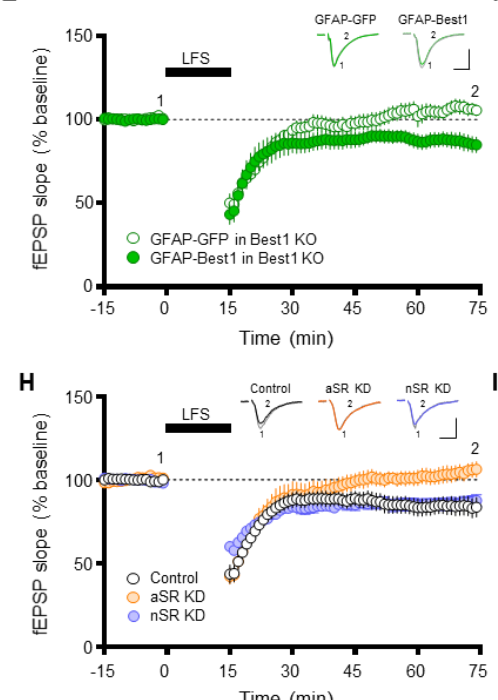

Time (min)

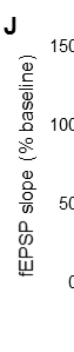

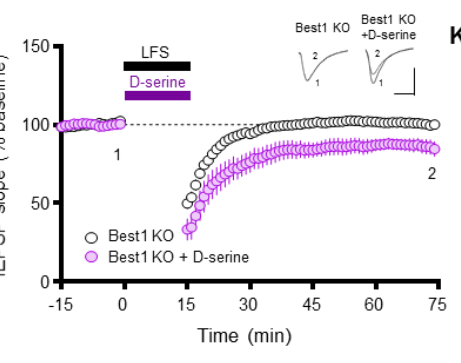
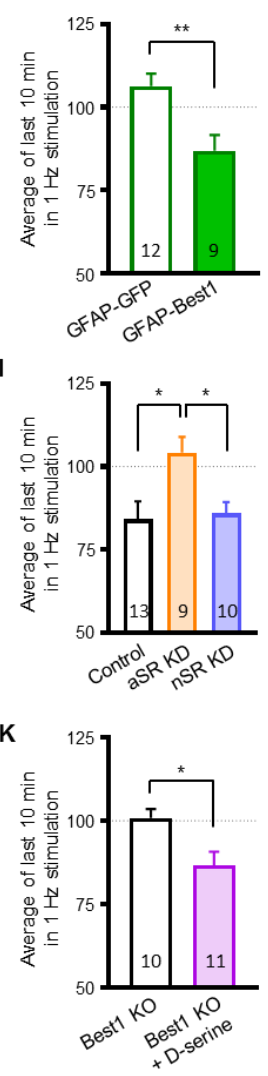

Figure 3. Astrocytic regulation of NMDAR tone through Best1 is important for the induction of LTD.

(A) LFS (900 stim. at $1 \mathrm{~Hz}$ )-induced LTD in WT and Best1 KO. (B)

Summary graph of LFS-induced

LTD in each condition. (C)

Bienenstock-Cooper-Munro (BCM) curve of synaptic plasticity in each condition. (D) Scheme of CA1 astrocyte-specific Best1 rescue in Best1 KO. (E and F) LFS-induced LTD $(E)$ and summary graph $(F)$ in CA1 astrocyte-specific Best1 rescue from Best1 KO. (G) Scheme of celltype specific SR knockdown. ( $\mathrm{H}$ and I) LFS-induced LTD $(\mathrm{H})$ and summary graph (I) in cell-type specific mSR knockdown experiment. ( $\mathrm{J}$ and $\mathrm{K}$ ) LTD induction in Best1 KO with D-serine application during LFS $(\mathrm{J})$ and summary graph $(\mathrm{K})$. Numbers in the graphs refer to hippocampal slices.

Data are represented as mean \pm SEM. ${ }^{*} p<0.05 ;{ }^{* *} p<0.01 ;{ }^{* * *} p<$ 0.001; unpaired t-test (B, C, F and $\mathrm{K})$, and one-way ANOVA with Tukey's multiple comparison test (I). 


\section{NE induces NMDAR tone and LTD in hippocampus}

224 It has been reported that exogenously applied NE by itself (without LFS) induces NMDAR-dependent

225 LTD (NE-LTD) through a1-AR (Scheiderer et al., 2004), which is predominantly expressed in astrocytes

226 (Hertz et al., 2010). To test if NE-LTD is caused by NMDAR tone, we first examined whether NE can

227 increase astrocytic $\mathrm{Ca}^{2+}$ through $\alpha 1-\mathrm{AR}$ and NMDAR tone via Best1 in the hippocampus. We expressed

228 jRCaMP1a, a genetically encoded $\mathrm{Ca}^{2+}$ indicator, in CA1 astrocytes with AAV-GFAP104-jRCaMP1a virus

229 (Figures 4A and 4B) and observed robust $\mathrm{Ca}^{2+}$ responses in astrocytes by $200 \mu \mathrm{M}$ NE application, which

230 were significantly blocked by prazosin, an a1-AR blocker (Figure 4C). These results indicate that NE

231 induces astrocytic $\mathrm{Ca}^{2+}$ through a1-AR, consistent with a previous report (Duffy and MacVicar, 1995). We

232 then recorded NE-induced exNMDAR current and found that NE caused a significant exNMDAR current

233 in CA1 pyramidal neuron, which was completely blocked by APV (Figures 4D and 4F). This NE-induced

234 exNMDAR current was almost completely eliminated in Best1 $\mathrm{KO}$, and significantly restored by D-serine

235 application (Figures 4E and 4F), indicating that NE increases NMDAR tone through Best1 in the

236 hippocampus. To investigate whether NE-LTD is mediated by NMDAR tone through Best1, we performed

237 fEPSP recordings in WT and Best1 KO, and found that NE-induced LTD was blocked by APV in WT

238 (Figures 4G and 4I), absent in Best1 KO (Figures 4H and 4I), and restored by D-serine in Best1 KO

239 (Figures $4 \mathrm{H}$ and $4 \mathrm{I}$ ). Taken together, these results indicate that NE activates astrocytic $\alpha 1-\mathrm{AR}$ to induce

240 LTD by increasing NMDAR tone through Best1. 

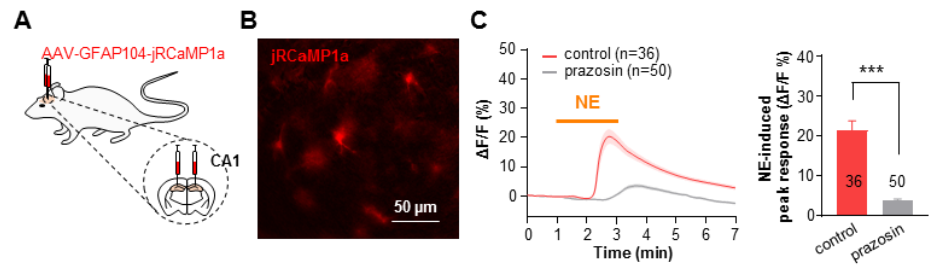

D

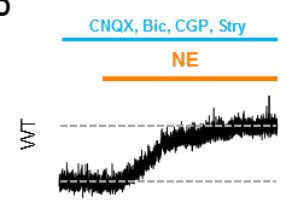

E
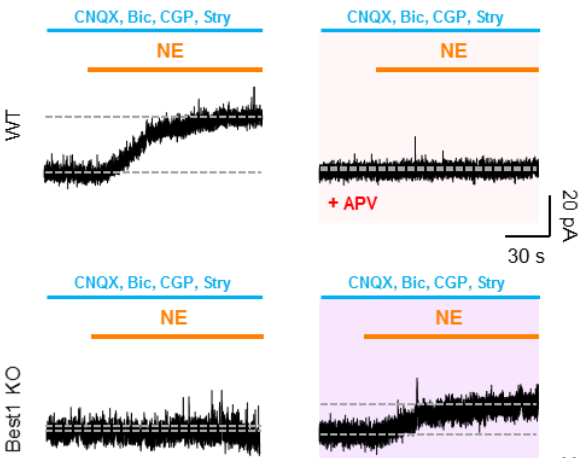

G

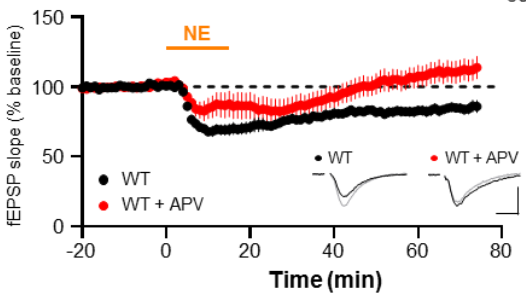

H

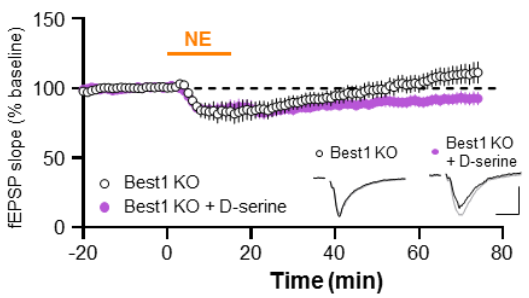

$\mathbf{F}$$$
\text { F }
$$
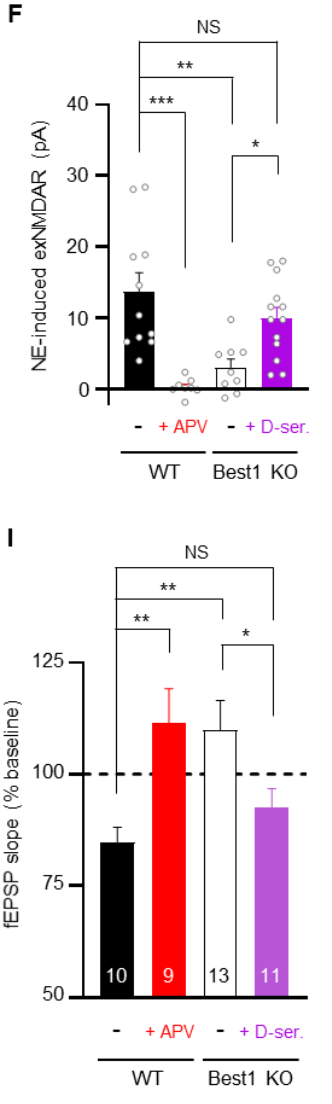

Figure 4. NE induces astrocytic $\mathrm{Ca}^{2+}$, NMDAR tone increase and NE-LTD.

(A) Astrocytic expression of jRCaMP1a with AAV-GFAP104-jRCaMP1a. (B) Fluorescent image of jRCaMP1a in hippocampal CA1. (C) Left, $200 \mu \mathrm{M}$ NE-induced jRCaMP1a response with or without $10 \mu \mathrm{M}$ prazosin, a1-AR blocker. Right, Summary of NE-induced peak response. (D) Representative traces of NEinduced exNMDAR current in WT with or without APV. (E) Representative traces of NEinduced exNMDAR current in Best1 $\mathrm{KO}$ with or without $100 \mu \mathrm{M} D$-serine. (F) Summary graph of NE-induced exNMDAR current in each condition. (G) NE-induced LTD in WT with or without APV. (H) NE-induced LTD in Best1 KO with or without $100 \mu \mathrm{M} D$-serine. (I) Summary graph of NE-induced LTD in each condition. Numbers in the graphs refer to cells (in C) or hippocampal slices (in I), and individual dots refer to cells. Data are represented as mean \pm SEM. ${ }^{*} p<0.05 ;{ }^{* *} p<0.01 ;{ }^{* * *} p<0.001$; Mann Whitney test (C), One-way ANOVA with Tukey's multiple comparisons test $(F)$, and unpaired t-test (I). 
241

242

243

244

245

246

247

248

249

250

251

252

253

254

255

256

257

258

259

260

261

262

\section{Endogenous NE is released via axo-axonic synapses to mediate heterosynaptic LTD}

The release of NE throughout the brain is critical for modulating arousal, attention, and cognitive behaviors, and its disruption is strongly associated with several psychiatric and neurodegenerative disorders in humans (Schwarz and Luo, 2015). Most of the NE released in the brain is supplied by the fiber projections from a very small, bilateral nucleus in the brainstem called the locus coeruleus. The local release of NE is reported to be stimulated by glutamate (Howells and Russell, 2008; Malva et al., 1994) or conventional electrical stimulation of Schaffer collateral pathway (Feng et al., 2019), raising a possibility that local NE release (Jacobowitz, 1979) is mediated by axo-axonic synapses in the hippocampus (Schwarz and Luo, 2015). Thus, we investigated the local NE release onto hippocampal astrocytes and its role for LTD. To visualize local NE release onto hippocampal astrocytes, GRABNE2m, a GPCR-based NE fluorescence sensor, was expressed in hippocampal astrocytes via injection of AAV-GFAP104-

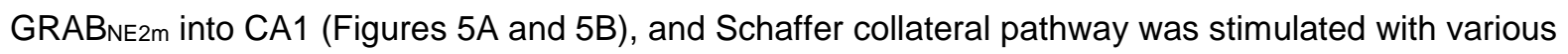
stimulation intensities and frequencies (Figures $5 \mathrm{C}$ and $5 \mathrm{D}$ ). We found that the electrical stimulation increased GRABNE2m fluorescence from astrocytes, in stimulation intensity- and frequency-dependent manners, with a peak response at $50 \mathrm{~Hz}$ (Figures 5E-5G). The evoked fluorescence was significantly blocked by Yohimbine (Figure $5 \mathrm{H}$ ), indicating that $\mathrm{NE}$ is released onto hippocampal astrocytes during the stimulation of Schaffer collateral pathway. To test whether glutamate contributes to the local NE release onto astrocytes, blockers of glutamate receptors were independently applied during the stimulation. We found that the NE response was majorly blocked by inhibition of AMPAR with CNQX, but minimally by inhibition of mGluR5 with MPEP or NMDAR with APV (Figures 5I-5K). These results indicate that stimulation of Schaffer collateral pathway induces local NE release by activating AMPAR, most likely localized at the axo-axonic presynaptic terminals of the LC projection fibers (Schwarz and Luo, 2015). 
264 only homosynaptic LTP but also heterosynaptic LTD (Scanziani et al., 1996). To investigate the role of

265 local NE release in homosynaptic LTP and heterosynaptic LTD, we performed simultaneous recordings of

266 homosynaptic and heterosynaptic fEPSP (Figure 5L). Two independent pathways were accessed with

267 theta micropipette as a bipolar microelectrode and validated by the lack of heterosynaptic paired-pulse

268 facilitation (Figure 5M). We observed a robust induction of heterosynaptic LTD while homosynaptic LTP

269 was induced by HFS (Figures 5N and 5O). Surprisingly, the heterosynaptic LTD, but not homosynaptic

270 LTP, was blocked by prazosin (Figures $5 \mathrm{~N}$ and 50), indicating that $\alpha 1-\mathrm{AR}$ activation is necessary for

271 heterosynaptic LTD. More importantly, we observed that the heterosynaptic LTD, but not homosynaptic

272 LTP, was impaired in Best1 KO (Figures 5P and 5Q). The impaired heterosynaptic LTD in Best1 KO was

273 fully restored by an enhancement of NMDAR tone with D-serine (Figures 5P and 5Q). Taken together,

274 these results indicate that local NE- $\alpha 1-A R$ signaling mediates heterosynaptic LTD through NMDAR tone. 
A
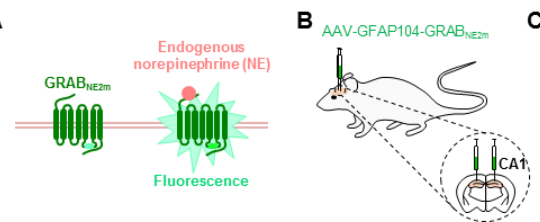

E
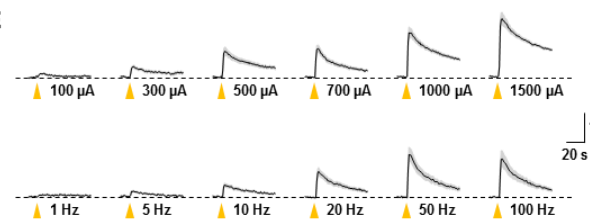

$\mathbf{F}$
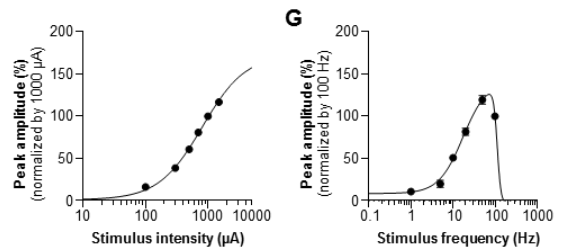

$\mathbf{L}$

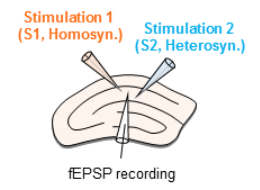

M

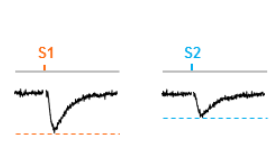

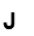

H

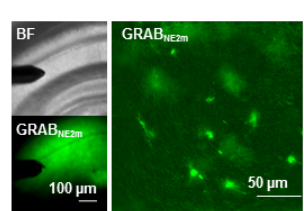

I

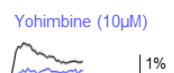

MPEP (10MM)
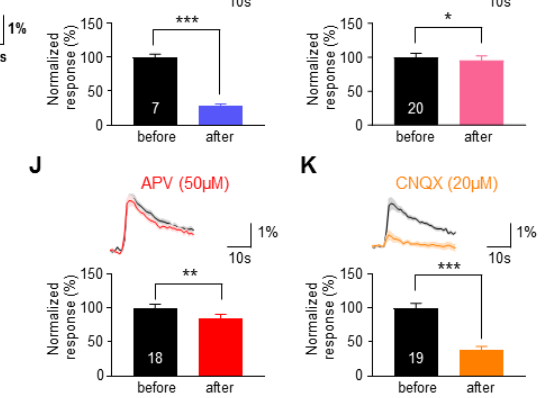

K
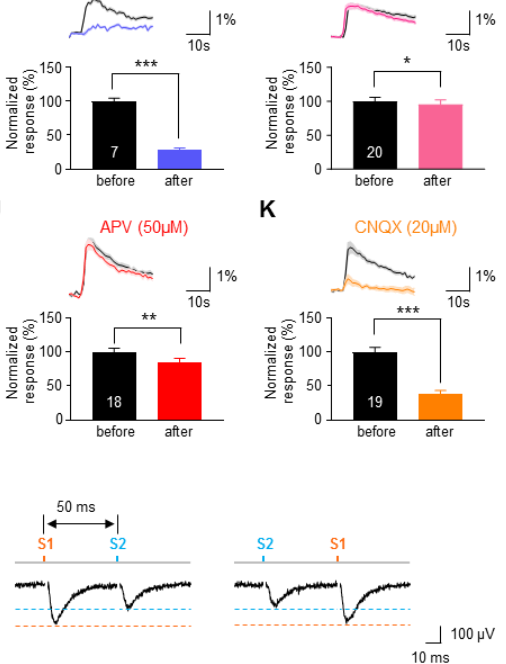

N

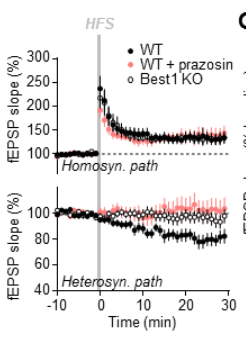

\begin{abstract}
○
\end{abstract}

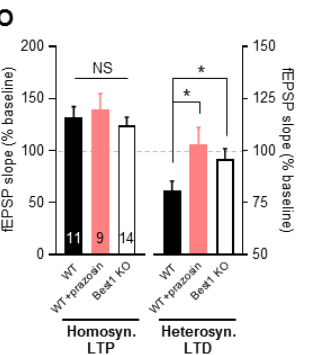

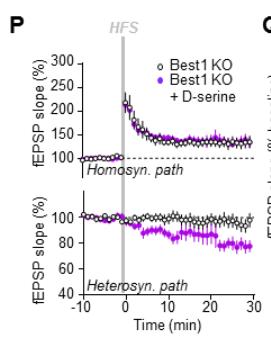

Q

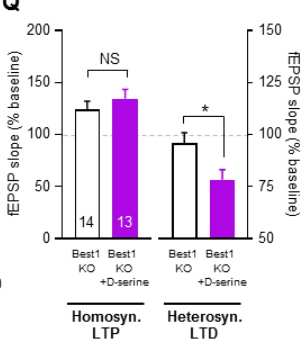

Figure 5. Endogenously released NE by glutamate induces heterosynaptic LTD through astrocytes.

(A) GRAB NE2m, a fluorescent sensor for NE. (B) Astrocytic expression of $\mathrm{GRAB}_{\mathrm{NE} 2 \mathrm{~m}}$ with AAV-GFAP104-GRAB ${ }_{\text {NE2m }}$. (C) Scheme of evoked NE release by Schaffer collaterals stimulation.

(D) Images of $\mathrm{GRAB}_{\mathrm{NE} 2 \mathrm{~m}}$ in hippocampal CA1. (E)

Representative traces of $\mathrm{GRAB}_{\mathrm{NE2m}}$ response by the various stimulation. $(F)$ Stimulus intensity-GRAB $\mathrm{BE}_{\mathrm{NE}}$ response curve (at $20 \mathrm{~Hz}$ ). (G) Stimulus frequency-GRAB $B_{N E 2 m}$ response curve (at $500 \mu \mathrm{A})$. (H-K) Inhibition of $\mathrm{GRAB}_{\mathrm{NE} 2 \mathrm{~m}}$ response by Yohimbine (H), MPEP (I), APV (J) and CNQX (K). (L) Scheme of simultaneous homosynaptic (S1, orange) and heterosynaptic (S2, blue) recordings. (M) Lack of heterosynaptic facilitation with 50 ms interval. (N) Homosynaptic and heterosynaptic changes by HFS in WT, WT with $10 \mu \mathrm{M}$

prazosin, and Best1 KO. (O) Summary graph of fEPSP changes in $(\mathrm{N})$. (P) Homosynaptic and heterosynaptic changes by HFS in Best1 KO and Best1 KO with $100 \mu \mathrm{M}$ D-serine. (Q) Summary graph of fEPSP changes in (P). Numbers in the graphs refer to hippocampal slices. Data are represented as mean \pm SEM. ${ }^{*} p<0.05 ;{ }^{* *} p<0.01$; ${ }^{* *} p<0.001$; unpaired t-test $(O$ and $Q)$ and paired t-test $(\mathrm{H}, \mathrm{I}, \mathrm{J}, \mathrm{K})$. 


\section{NMDAR tone-dependent heterosynaptic LTD is required for repotentiation LTP}

276 Heterosynaptic plasticity has been proposed to enable further changes in synaptic plasticity, i.e.,

277 metaplasticity (Chen et al., 2013b). Given that Best1 KO showed the lack of heterosynaptic LTD, we

278 examined further changes in synaptic plasticity after HFS in Best1 KO. To test a bidirectional modification

279 of metaplasticity after the $1^{\text {st }} \mathrm{HFS}$ potentiation LTP, we delivered additional LFS and $2^{\text {nd }} \mathrm{HFS}$ during

280 fEPSP recordings (Figure 6A), as previously described (Dudek and Bear, 1993). We found that the LFS-

281 induced depotentiation LTD was observed in both WT and Best1 KO (Figures 6A and 6B). In contrast, the

$2822^{\text {nd }}$ HFS-induced repotentiation LTP was significantly impaired in Best1 KO (Figures 6A and 6B). This

283 pattern of intact depotentiation LTD and impaired repotentiation LTP in Best1 KO was similarly observed

284 when LTP-inducing stimulation was changed from HFS to theta-burst stimulation (TBS) (Figures S5E and

285 S5F). These results indicate that the lack of NMDAR tone and heterosynaptic LTD in Best1 KO leads to

286 the impaired repotentiation LTP. To test whether the impaired repotentiation LTP in Best1 KO can be

287 restored by an enhancement of NMDAR tone during each stimulation period, we applied D-serine during

$2881^{\text {st }}$ HFS potentiation (orange), LFS (green), or $2^{\text {nd }}$ HFS repotentiation (blue) (Figures $6 \mathrm{C}$ and $\left.6 \mathrm{E}\right)$. We

289 found that the impaired repotentiation LTP in Best1 KO was fully restored by D-serine treatment only

290 during $1^{\text {st }} \mathrm{HFS}$ potentiation (Figures $6 \mathrm{C}$ and $6 \mathrm{D}$ ), but not during LFS or $2^{\text {nd }} \mathrm{HFS}$ repotentiation (Figures $6 \mathrm{E}$

291 and 6F). These results indicate that NMDAR tone-dependent heterosynaptic LTD is critical for

292 subsequent repotentiation LTP and metaplasticity. 
bioRxiv preprint doi: https://doi.org/10.1101/2021.03.25.436945; this version posted March 25, 2021. The copyright holder for this preprint (which was not certified by peer review) is the author/funder. All rights reserved. No reuse allowed without permission.

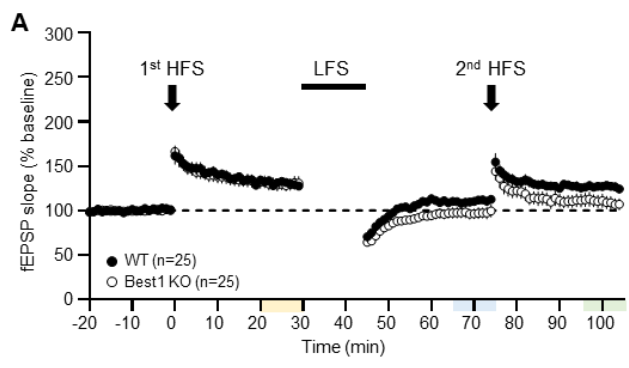

\section{B}
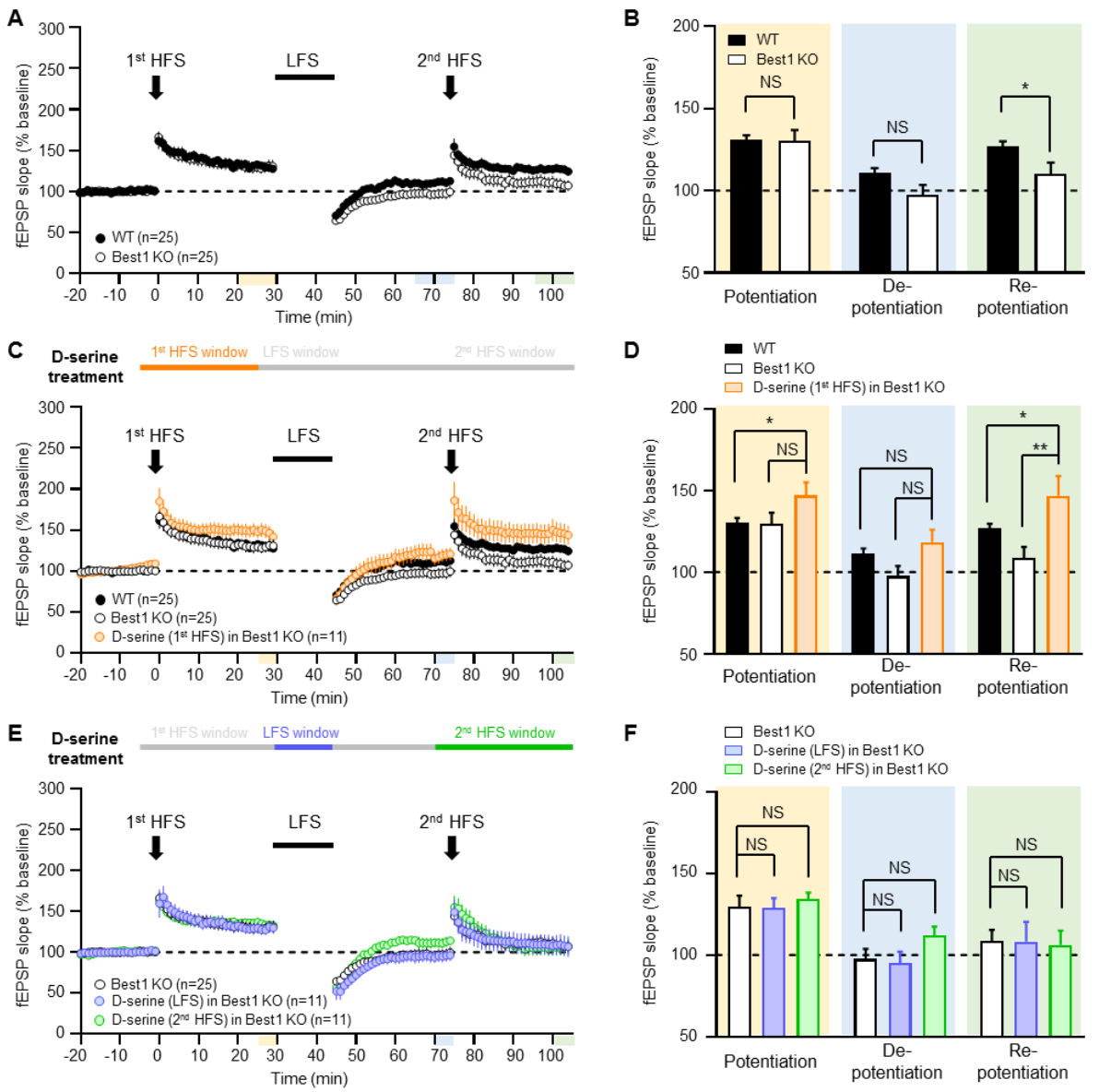

D

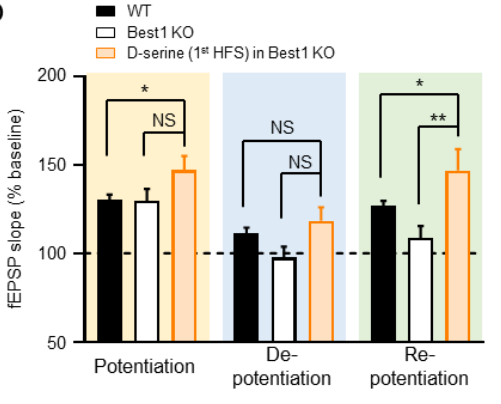

F $\square$ Best1 Ko

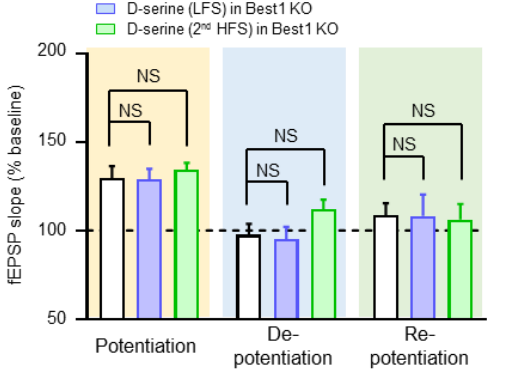

Figure 6. NMDAR tone during first potentiation facilitate repotentiation.

( $A$ and $B$ ) Time course of the normalized fEPSP slope changes $(A)$ and summary graph (B) of $1^{\text {st }}$ HFS-induced potentiation, LFS-induced depotentiation and $2^{\text {nd }} \mathrm{HFS}$ induced repotentiation in WT and Best1 KO. (C) NMDAR tone enhancement during $1^{\text {st }}$ HFS window in Best $1 \mathrm{KO}$ by 20 $\mu \mathrm{M}$ D-serine treatment. (D) Summary graph of results from (C). (E) NMDAR tone enhancement during LFS or $2^{\text {nd }}$ HFS window in Best $1 \mathrm{KO}$ by 20 $\mu \mathrm{M}$ D-serine treatment. (F) Summary graph of the results from (E). Data are represented as mean \pm SEM. ${ }^{*} p<0.05 ;{ }^{* *} p$ $<0.01$; ${ }^{* * *} p<0.001$; unpaired $t-$ test (B, $D$ and $F)$. 


\section{Spatial reversal learning is impaired in Best1 $\mathrm{KO}$ and rescued by astrocytic Best1}

294 To investigate the role of NMDAR tone-dependent heterosynaptic LTD in learning and memory, we

295 performed various hippocampus-dependent memory tasks such as Morris water maze (MWM) test,

296 passive avoidance test (PAT) and contextual fear conditioning (CFC) test with WT and Best1 KO (Figures

297 7A and S6B-S6E). We found that in MWM, PAT, and CFC, there was no difference in memory acquisition

298 between WT and Best1 KO (Figure 7B and Figures S6B-S6E). In contrast, we observed that Best1 KO

299 showed significantly impaired spatial reversal learning when the hidden platform was relocated at

300 opposite quadrant $(\mathrm{O})$ during the reversal task session (Figures 7B and 7C); Best1 $\mathrm{KO}$ spent significantly

301 more time in the original target quadrant $(\mathrm{O})$ and significantly less time in T, compared to WT (Figure 7C).

302 This impaired spatial reversal learning in Best1 $\mathrm{KO}$ was not due to a malfunction of vision or locomotion,

303 as evidenced by an intact acquisition when we switched from the hidden to a visible platform (Figures 7D,

304 7E and S6A). These results imply that Best1-dependent NMDAR tone and heterosynaptic LTD are

305 necessary for spatial reversal learning and flexible memory. To further test whether astrocyte-specific

306 Best1 rescue sufficiently restores spatial reversal learning in Best1 KO, we injected AAV-GFAP-Best1-

307 IRES-EGFP virus bilaterally into CA1 hippocampus of Best1 KO and performed MWM test. We found a

308 significant restoration of spatial reversal learning in Best1 KO (Figure 7F), indicating that CA1-astrocyte-

309 specific Best1 rescue is sufficient for spatial reversal learning and flexible memory. Taken together, these

310 results establish a causal relationship between the astrocytic Best1 in CA1 hippocampus and spatial

311 reversal learning and flexible memory.

312

313 NMDAR tone increase during memory acquisition is critical for flexible memory 
314 Although LFS-induced homosynaptic LTD has been implicated in spatial reversal learning (Dong et

315 al., 2013; Nicholls et al., 2008), there have been conflicting results (Rutten et al., 2011; Zhang and Wang,

316 2013), raising a possibility that heterosynaptic, not homosynaptic, LTD might be responsible for spatial

317 reversal learning. Considering the fact that heterosynaptic LTD occurs at the time of homosynaptic LTP,

318 we hypothesized that heterosynaptic LTD during the initial memory acquisition contributes to spatial

319 reversal learning and flexible memory, as it enables further repotentiation LTP (Figures 6C and 6D). To

320 test this hypothesis, Best1 $\mathrm{KO}$ mice were injected with D-serine (600 mg/kg, intra-peritoneal) during the

321 initial memory acquisition session in MWM (Figures 7G-7L). We found that application of D-serine during

322 the initial memory acquisition session fully restored the impaired spatial reversal learning in Best1 $\mathrm{KO}$

323 (Figures $7 \mathrm{~K}$ and $7 \mathrm{~L}$ ). These results imply that heterosynaptic LTD during the initial memory acquisition is

324 critical for the formation of flexible memory (Figures S7A-S7D). Altogether, these findings establish that

325 astrocytes render memory flexible through regulation of Best1-dependent NMDAR tone and

326 heterosynaptic LTD during the initial stage of memory acquisition. 


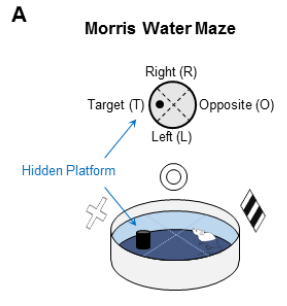

D

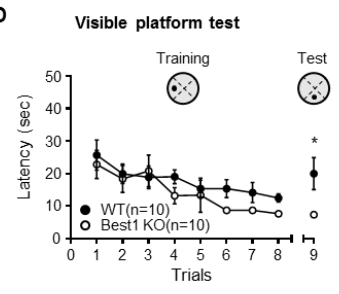

B

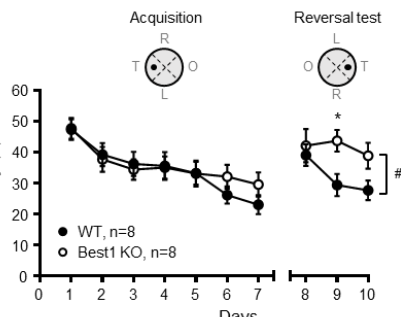

E

E

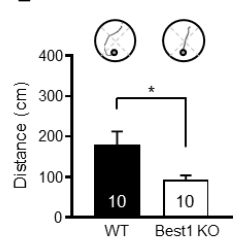

F b-CTI: Astrocyic Best rescue Best KO CA1
CAM

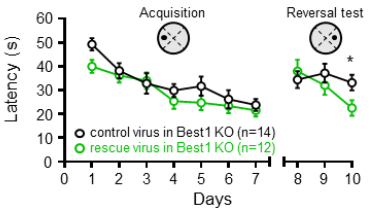

G

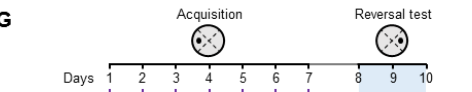

Acquisition
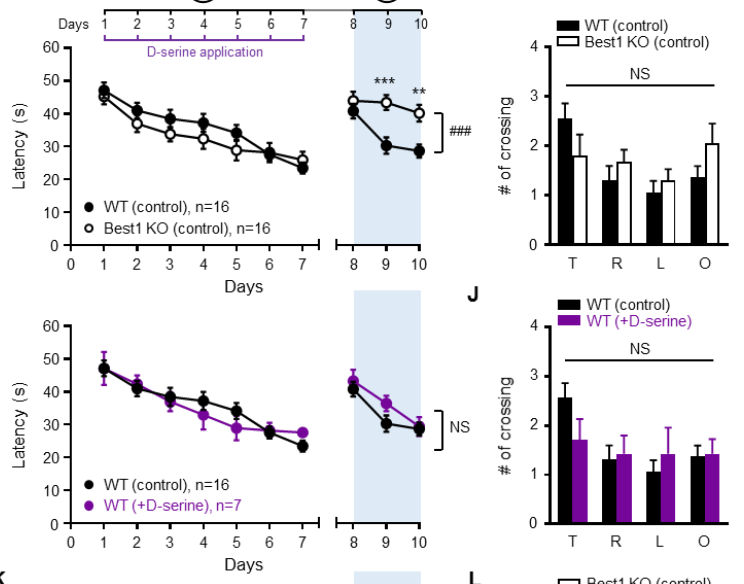

K

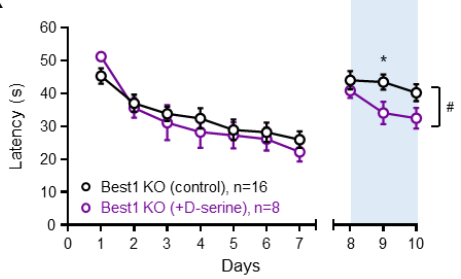

L ${ }_{4}^{\text {Best1 KO (control) }}$

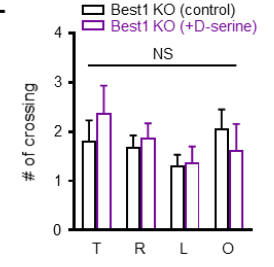

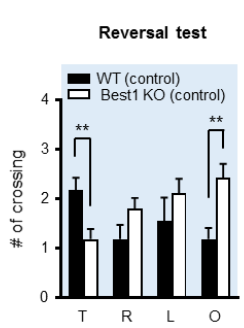

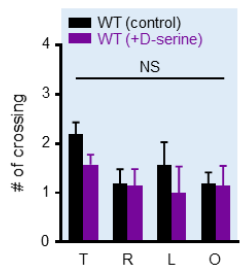

C
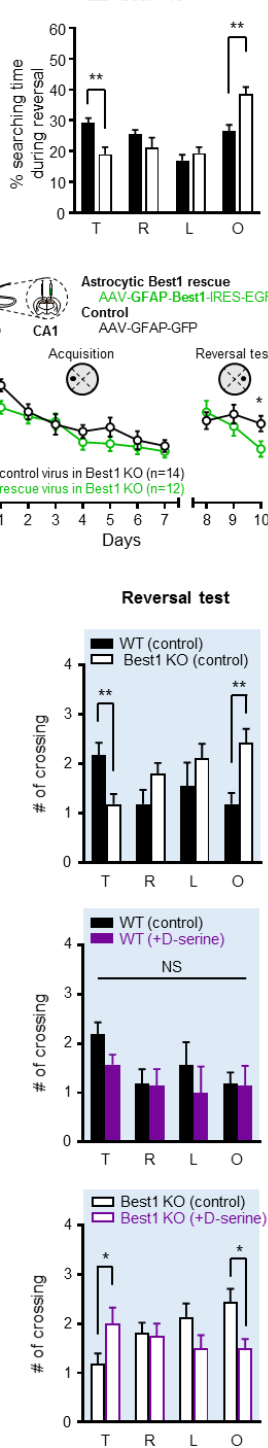

Figure 7. NMDAR tone is critical for formation of flexible memory.

(A) Scheme of Morris water maze. (B)

Escape latency of WT and Best1 KO in acquisition and reversal test session. (C)

Searching time of WT and Best1 KO

during reversal test in each quadrant. (D and E) Escape latency (D) and moved distance $(\mathrm{E})$ of WT and Best1 $\mathrm{KO}$ in visible platform test. (F) Escape latency of Best $1 \mathrm{KO}$ with the rescue of astrocytic Best1, in acquisition and reversal test session during hidden platform test. (G-L) Application of saline or D-serine (600 $\mathrm{mg} / \mathrm{kg}$ ) in each day of acquisition session. (G), (I), (K) Escape latency of WT and Best1 KO with application of saline or D-serine. $(H),(J),(L)$ Left: number of crossing to each quadrant in each condition during acquisition session. Right: number of crossing to each quadrant in each condition during reversal test session. Numbers in the graphs refer to animals. Data are represented as mean \pm SEM. ${ }^{*} p<0.05$; ${ }^{* *} p<0.01 ;{ }^{* * *} p<0.001$; Two-way ANOVA with Fisher's LSD (B, D, F) and unpaired t-test (C, E, G-L). \#p < 0.05; \#\#p $<0.01$; \#\#\#p < 0.001; Two-way repeated measures (RM) ANOVA (genotype or treatment) (B, G, I, K). 


\section{Discussion}

In the present study, we have demonstrated for the first time that astrocytes are critically involved in reversal learning and flexible memory. Astrocytes achieve this unique function by co-releasing D-serine and glutamate through the $\mathrm{Ca}^{2+}$-activated anion channel Best1 upon activation of NE- $\mathrm{\alpha} 1-\mathrm{AR}$ signaling pathway, leading to an enhanced NMDAR tone and induction of heterosynaptic LTD during the initial period of memory acquisition (Figure S7A). The presence of astrocyte-driven NMDAR tone provides the molecular basis for both the formation of a flexible memory at the time of initial memory acquisition and the induction of repotentiation LTP and a new memory at the time of reversal learning (Figure S7B). In the absence of such astrocyte-derived NMDAR tone as in Best1 $\mathrm{KO}$ mice, the animals exhibited an impaired heterosynaptic LTD, impaired metaplasticity (i.e., repotentiation LTP), impaired reversal learning and flexible memory (i.e., a persistence of initial memory) (Figure S7C), which were all restored by a D-serine supplement or the astrocyte-specific reconstitution of Best1 in Best1 KO mice (Figure S7D). The impaired reversal learning and flexible memory are reminiscent of the major symptoms of ASD and schizophrenia, both of which share the common mechanism of NMDAR hypofunction. The unexpected role of NE and the astrocytic $\alpha 1-A R$ in the induction of heterosynaptic LTD is not surprising, considering the fact that NE is critical for attention, focus, and arousal. Our research will provide a comprehensive understanding of NE, NMDAR tone, and memory formation.

\section{Astrocyte as a regulator of NMDAR tone}

Since the first discovery of tonic activation of exNMDAR in CA1 pyramidal neurons (Sah et al., 1989), many independent research groups have investigated the molecular and cellular sources and functions of the tonic activation of exNMDAR using various nomenclatures such as "tonic NMDAR" and "NMDAR 
tone" (Parsons and Raymond, 2014). Although the two names may sound indistinguishable, they have profoundly different implications if one considers the properties of NMDAR. For a channel activation of exNMDAR, glutamate and co-agonist (glycine or D-serine) have to bind to their respective binding site at exNMDAR (Traynelis et al., 2010). However, the binding of two agonists is not sufficient for tonic activation of exNMDAR because of the $\mathrm{Mg}^{2+}$ block near resting membrane potential. The glutamate-andco-agonist-bound exNMDAR is activated only when a depolarization relieves the $\mathrm{Mg}^{2+}$ block (Sah et al., 1989). This is in great contrast to the extrasynaptic $G A B A_{A}$ receptors $\left(G A B A_{A} R\right)$, in which the receptors are tonically activated voltage-independently upon the binding of GABA (Egawa and Fukuda, 2013).

357 Thus, it is appropriate to name it, "tonic GABAAR." In contrast, for the exNMDAR in which glutamate and co-agonist are bound but not active under resting membrane potential, it is more appropriate to use the term "NMDAR tone", rather than "tonic NMDAR", as we did in the present study. 2007). However, this concept of astrocytic contribution to NMDAR tone has been challenged by the report

362 that knockout of IP3R2 (type 2 inositol 1,4,5-trisphosphate receptor), which is known to mediate endoplasmic reticulum (ER) $\mathrm{Ca}^{2+}$ release exclusively in astrocytes (Holtzclaw et al., 2002), showed no difference in hippocampal NMDAR tone (Petravicz et al., 2008). Contrary to this conflicting observation, we have found that astrocytes majorly contribute to the hippocampal NMDAR tone in a $\mathrm{Ca}^{2+}$-dependent manner through the $\mathrm{Ca}^{2+}$-activated anion channel Best1 (Figure 1). Our results raise a possibility that

367 IP3R2-mediated $\mathrm{Ca}^{2+}$ signal may not be necessary for the activation of Best1. In support of this possibility, IP3R2-independent $\mathrm{Ca}^{2+}$ release from ER has been reported (Okubo et al., 2018), and, more importantly, ER-Ca ${ }^{2+-i n d e p e n d e n t ~} \mathrm{Ca}^{2+}$ signal is found in astrocytic fine processes (Rungta et al., 2016; 
371 spotty $\mathrm{Ca}^{2+}$ from TRPA1) (Oh et al., 2019; Shigetomi et al., 2013) could also contribute to the activation of

372 Best1, and should be investigated in the future studies.

373 In a series of previous studies, we have demonstrated that 1) Best1 is permeable to glutamate and

374 mediates astrocytic glutamate release upon activation of PAR1 (Oh et al., 2012; Park et al., 2015; Park et

375 al., 2013; Park et al., 2009; Woo et al., 2012), 2) PAR1-driven astrocytic glutamate release targets

376 postsynaptic NMDARs, and 3) modulates hippocampal synaptic plasticity by lowering the threshold for

377 LTP (Lee et al., 2007; Park et al., 2015). In the current study, we have further identified D-serine as a

378 novel permeant anion passing through Best1 (Figure 2), and demonstrated that astrocytic Best1 is an

379 ideal regulator of NMDAR tone by releasing both glutamate and D-serine in hippocampus. Our study

380 directly addresses the recent controversy over the origin of D-serine (Papouin et al., 2017; Wolosker et

381 al., 2017) and provides answers by the astrocyte-specific manipulation of D-serine release through over-

382 expression of Best1 in astrocytes of the Best1 KO mice. Our results are consistent with the previous

383 reports that 1) D-serine affects not only LTP, but also LTD induction (Zhang et al., 2008), and 2) inhibition

384 of the mechanism mediating neuronal D-serine release does not affect LTD (Sason et al., 2017).

385 Additionally, utilizing the cell-type specific expression of SR shRNA, we have demonstrated that D-serine

386 synthesis from astrocytes, but not neurons, is necessary for LTD induction (Figure 3). These results

387 indicate that both co-release of D-serine and glutamate from astrocyte through Best1 and D-serine

388 synthesis by astrocytic SR are critical for hippocampal LTD.

389 Interestingly, we have found that D-serine administration alone was able to restore the impairments of

390 Best1 KO. These results appear to underrate the role of Best1-mediated glutamate release in the

391 hippocampus. However, under physiological condition Best1-mediated glutamate also contributes to

392 NMDAR tone (Figure 1), and is expected to play an important role when PAR1 is activated (Park et al., 
2015; Price et al., 2021). Indeed, we could observe that ambient glutamate was decreased by $37.4 \%$ in

Best1 $\mathrm{KO}$ compared to WT (Figure 1M), implying that there is a substantial remaining portion of ambient

glutamate. These results suggest that, in addition to Best1-mediated tonic glutamate release, there may

to NMDAR tone. These exciting possibilities await future investigations.

401 As astrocytes are heterogeneous in different brain regions (Khakh and Sofroniew, 2015), how the regulatory mechanism of NMDAR tone or intracellular metabolites differ from one brain region to another should be further investigated to reveal differential roles of NMDAR tone in different brain regions. It

404 should be noted that Best1 mediates glutamate and D-serine release in the cortex as well (Lalo et al., 405 2021), suggesting that it may also mediate NMDAR tone in medial prefrontal cortex (mPFC) (Povysheva 406 and Johnson, 2012) or other brain regions. Consequently, future researches on different regulatory 407 mechanisms in various brain regions will greatly broaden our understanding of the critical roles of $408 \quad$ NMDAR tone.

410 Local NE release mediates heterosynaptic LTD via astrocytic regulation of NMDAR tone

411 Activation of LC has been implicated in arousal, attention, and cognitive behaviors (Schwarz and Luo, 412 2015). However, since activation of LC exerts effects on a large area throughout the brain (Zerbi et al., 413 2019), an alternative mechanism is required when NE is utilized in local area. In this study, we have 
AMPAR (Ghersi et al., 2003) at the LC terminals of so-called en passant varicosities (Atzori et al., 2016) as in the form of axo-axonic synapses. These results support the previously proposed hypothesis for the

417 local control of NE release (Jacobowitz, 1979), and are consistent with the results that glutamate

418 stimulates local NE release (Howells and Russell, 2008; Malva et al., 1994). This local NE release can be

419 the basis of the recently proposed "glutamate amplifies noradrenergic effects" (GANE) model (Mather et

420 al., 2016). Considering the previous report that NE-induced NMDAR-dependent LTD is independent of

421 LFS-induced LTD (Scheiderer et al., 2004), the finding that NE release is prominent only at high

422 frequency, rather than low frequency, stimulation of the Schaffer collateral fibers from the CA3

423 glutamatergic neurons is not unexpected. High-frequency stimulation of Schaffer collateral fibers induces

424 homosynaptic LTP at stimulated synapses, and simultaneously releases NE to turn-on the cascade of

425 events of 1) activating astrocytic $\alpha 1-A R, 2)$ increase in NMDAR tone, and 3) induction of heterosynaptic

426 LTD at unstimulated synapses (Figures 4 and 5). These findings are consistent with the previous reports

427 that astrocytes play an essential role in heterosynaptic LTD (Chen et al., 2013a; Serrano et al., 2006).

428 Our study demonstrates that activation of astrocytes by local NE release subsequently enables the

429 plasticity of neighboring synapses (Figure 6), which goes in line with the concept of astrocytes

430 orchestrating synaptic dynamics (De Pitta et al., 2016). Given the fact that one astrocyte is in contact with

431 about 140,000 synapses from numerous neurons in CA1 of the adult rat (Bushong et al., 2002), it is

432 plausible to consider one astrocyte to mediate heterosynaptic LTD at the unstimulated synapses while the

433 stimulated synapses are potentiated. Thus, astrocytes provide a unique structural medium for a

434 simultaneous dynamic control of multiple synapses, from both stimulated and unstimulated neurons,

435 mediating various forms of homeostatic plasticity and metaplasticity. This notion is also supported by the

436 results of accompanying paper (Lalo et al., 2021). 


\section{Heterosynaptic LTD determines flexibility of memory}

439 Our study attempts to fill in the huge gap between the inadequately simple concept of homosynaptic

440 plasticity and the complex nature of memory formation, retention, and flexibility. Cognitive flexibility has

441 long been explained only by homosynaptic LTD (Dong et al., 2013; Kim et al., 2011; Nicholls et al., 2008).

442 However, because several studies have shown that homosynaptic LTD is not indispensable for cognitive

443 flexibility (Rutten et al., 2011; Zhang and Wang, 2013), an alternative mechanism of synaptic plasticity for

444 cognitive flexibility has been needed. In the present study, we have demonstrated for the first time that

445 heterosynaptic LTD accompanying homosynaptic LTP contributes to cognitive flexibility. The biggest

446 difference in the function between homosynaptic and heterosynaptic LTD for cognitive flexibility is that

447 homosynaptic LTD acts when memory modification is required (Dong et al., 2013), whereas

448 heterosynaptic LTD occurs during memory acquisition. This novel concept is supported by the

449 observation that impaired reversal learning was restored by increasing the NMDAR tone of Best1 KO

450 mice during the initial memory formation (Figure 7). We interpret these results as when the initial memory

451 is formed, the memory that accompanies heterosynaptic LTD becomes "flexible memory", and the

452 memory that does not accompany becomes "inflexible memory". This novel idea predicts that less-flexible

453 memory can be formed during memory acquisition under certain conditions in which heterosynaptic LTD

454 is impaired. In support of this prediction, prazosin administered during threat memory formation in mice

455 and humans has been reported to interfere with subsequent extinction learning (Do-Monte et al., 2010;

456 Homan et al., 2017), which is consistent with our observation that impaired heterosynaptic LTD interferes

457 subsequent learning (Figure 7). The decrease in glutamate-induced NE release during aging (Dezfuli et

458 al., 2019) also suggests that declined cognitive flexibility in the elderly (Boone et al., 1993) can also be 
459 due to impaired NE- $\alpha 1-A R-d e p e n d e n t$ heterosynaptic LTD. Similarly, as LC is one of the most vulnerable

460 regions in the progression of $A D$ (Matchett et al., 2021), declined cognitive flexibility in the early stages of

461 AD (Guarino et al., 2018) can be resulted from the impaired heterosynaptic LTD. In addition to AD,

462 although degeneration of LC in ASD and schizophrenia was found to be minimal (Craven et al., 2005;

463 Martchek et al., 2006), the impaired cognitive flexibility is possibly due to a decreased local NE release or

464 NMDAR hypofunction. Altogether, our findings extend our current knowledge of reversal learning and

465 behavioral flexibility beyond synaptic plasticity to the novel concepts of heterosynaptic LTD, repotentiation

466 LTP, and flexible memory.

467 In conclusion, we have established that astrocytes play a crucial role in forming a flexible memory by

468 enabling heterosynaptic LTD at unstimulated synapses, such that a new memory is easily formed when

469 environment and circumstances change. These findings broaden our understanding of astrocytic roles in

470 memory formation, and provide potential therapeutic targets for impaired cognitive flexibility in various

471 psychiatric diseases. 


\section{Acknowledgments}

473 This study was supported by the Creative Research Initiative Program funded by National Research

474 Foundation (NRF) of Korea (2015R1A3A2066619), Korea Institute of Science and Technology

475 Institutional Program (project no. 2E26860), and Institute for Basic Science (IBS), Center for Cognition

476 and Sociality (IBSR001-D2) to C.J.L. Thanks to Dr.Yuriy Pankratov for proofreading the manuscript.

\section{$478 \quad$ Author Contributions}

479 W Koh, YE Chun, J Lee, MG Park, H Kang, J Woo, H Chun performed electrophysiological experiments.

480 W Koh, M Park, HS Shim performed behavioral experiments. W Koh and S Kim performed slice imaging

481 experiments. W Koh, MG Park, SJ Oh, S Lee, J Hong, J Feng performed molecular experiments. Y Li, H

482 Ryu, J Cho, and CJ Lee gave technical support and conceptual advice. CJ Lee supervised the project. W

483 Koh and CJ Lee wrote the manuscript.

\section{Declaration of Interests}

486 The authors declare no competing interests. 
487

\section{STAR $\star$ METHODS}

\section{Contact for Reagent and Resource Sharing}

Further information and requests for reagents may be directed to, and will be fulfilled by the corresponding author, C. Justin Lee (cjl@ibs.re.kr).

\section{Animals}

Mice were given ad libitum access to food and water and were kept under a 12:12-h light-dark cycle. All animals were housed in groups of 3-5 per cage. All animal care and handling was performed according to the directives of the Institutional Animal Care and Use Committee of Korea Institute of Science and Technology (Seoul, South Korea) and of Institute for Basic Science (Daejeon, South Korea).

\section{Stereotaxic virus injection into hippocampal CA1}

Viruses used in this study were produced from Korea Institute of Science and Technology (KIST) Virus Facility (Seoul, South Korea) or Institute for Basic Science (IBS) virus facility (Daejeon, South Korea). Mice were anesthetized with isoflurane and mounted into stereotaxic frames (David Kopf Instruments, Tujunga, CA, USA). Viruses were bilaterally injected using syringe pump (KD Scientific, Holliston, MA, USA) into CA1 of hippocampus with the following coordinates (from bregma): anterior-posterior, $-1.8 \mathrm{~mm}$; medial-lateral, $\pm 1.5 \mathrm{~mm}$, dorsal-ventral, $\pm 1.7 \mathrm{~mm}$.

\section{Preparation of brain slice for the electrophysiological and imaging experiments}

Brain slice were prepared as previously performed (Lee et al., 2007). Briefly, mice were anaesthetized with isoflurane and decapitated to isolate the brain. The brain was excised after decapitation, cut into 350$\mu \mathrm{m}$-thick transverse or coronal slices using vibrating microtome (DSK Linearslicer ${ }^{\mathrm{TM}}$ Pro7, DSK, Japan) in ice-cold, oxygenated $\left(95 \% \mathrm{O}_{2} / 5 \% \mathrm{CO}_{2}\right)$ sucrose-based dissection buffer containing $5 \mathrm{KCl}, 1.23 \mathrm{NaH}_{2} \mathrm{PO}_{4}$, $26 \mathrm{NaHCO}_{3}, 10$ glucose, $0.5 \mathrm{CaCl}_{2}, 10 \mathrm{MgSO}_{4}$, and 212.5 sucrose (in $\mathrm{mM}$ ). Brain slices were left to recover for at least 1 hour before recording, and were used for whole-cell patch recording and imaging experiments.

For the fEPSP experiment, 400- $\mu \mathrm{m}$-thick transverse of hippocampal slices were prepared and incubated in oxygenated artificial cerebrospinal fluid (aCSF) containing $124 \mathrm{NaCl}, 5 \mathrm{KCl}, 1.25 \mathrm{NaH}_{2} \mathrm{PO}_{4}, 2.5 \mathrm{CaCl}_{2}$, $1.5 \mathrm{MgCl}_{2}, 26 \mathrm{NaHCO}_{3}$ and 10 dextrose (in $\mathrm{mM}$ ) at $28 \pm 1^{\circ} \mathrm{C}$ for at least 1 hour.

\section{Whole-cell patch recording experiments}

Whole-cell patch recordings were performed under the standard aCSF recording solution (130 $\mathrm{NaCl}, 24$ $\mathrm{NaHCO}_{3}, 3.5 \mathrm{KCl}, 1.25 \mathrm{NaH}_{2} \mathrm{PO}_{4}, 1.5 \mathrm{CaCl}_{2}, 1.5 \mathrm{MgCl}_{2}$ and 10 glucose (in mM)) saturated with $95 \% \mathrm{O}_{2}$ and $5 \% \mathrm{CO}_{2}$. Patch pipette electrodes $(4-7 \mathrm{M} \Omega$ ) were fabricated from borosilicate glass (GC150F-10, Warner Instrument Corp., USA). 
To measure exNMDAR current, recording electrodes were filled with an internal solution containing 135 $\mathrm{CsMeSO}_{4}, 8 \mathrm{NaCl}, 0.25$ EGTA, 10 HEPES, 4 Mg-ATP, $0.3 \mathrm{Na} 2-\mathrm{GTP}$, and $5 \mathrm{QX} 314$ (in mM) (pH adjusted to 7.3 with $\mathrm{CsOH}$ ). Baseline current was stabilized under treatment of CNQX (20 $\mu \mathrm{M})$, Bicuculline (10 $\mu \mathrm{M})$, CGP $55845(10 \mu \mathrm{M})$, and Strychnine $(10 \mu \mathrm{M})$. The amplitude of exNMDAR current was measured by the baseline shift after $50 \mu \mathrm{M}$ APV application. Signals were amplified using MultiClamp 700B (Molecular Devices, USA), and data was acquired and analyzed using a Digitizer 1550B (Molecular Devices, USA) and pClamp software (Molecular Devices, USA), respectively. To block $\mathrm{Ca}^{2+}$ signal in hippocampal astrocytes using BAPTA, patch electrodes were filled with an internal solution containing $123 \mathrm{CsCl}, 1$ $\mathrm{MgSO}_{4}, 10$ HEPES, 10 BAPTA, 100 Alexa fluor 488 hydrazide, $4 \mathrm{Mg}$-ATP, and $0.3 \mathrm{Na}$-GTP (in mM) (pH adjusted to 7.35 with $\mathrm{CsOH}$, and osmolality adjusted to $282 \mathrm{mOsmol} / \mathrm{kg}$ ), as previously performed (refer). To measure evoked EPSC (eEPSC) and NMDA/AMPA, recording electrodes were filled with an internal solution containing $140 \mathrm{CsMeSO}_{4}, 8 \mathrm{NaCl}, 1 \mathrm{MgCl}_{2}$, $0.5 \mathrm{EGTA}, 10 \mathrm{HEPES}, 7$ phosphocreatine di(tris) salt, $4 \mathrm{Mg}$-ATP, $0.3 \mathrm{Na}_{2}$-GTP, and $5 \mathrm{QX} 314$ (in mM) (pH adjusted to 7.3 with NMDG). Whole-cell voltageclamp recordings were made from CA1 pyramidal neurons, and Schaffer collateral pathway was stimulated using a concentric bipolar electrode (CBBPE75, FHC, Bowdoin, ME, USA). AMPA-mediated current was recorded with holding at $-60 \mathrm{mV}$ and NMDA-mediated current was recorded with holding at $+40 \mathrm{mV}$ in the presence of CNQX. Stimulus intensity was adjusted to evoke an eEPSC of approximately $30-40 \%$ of the maximal amplitude.

541 To perform LTD and metaplasticity experiment, recording electrodes were filled with an internal solution containing $140 \mathrm{CsMeSO}_{4}, 8 \mathrm{NaCl}, 1 \mathrm{MgCl}_{2}$, $0.05 \mathrm{EGTA}, 0.0244 \mathrm{CaCl}_{2}, 10 \mathrm{HEPES}, 7$ phosphocreatine di(tris) salt, 4 Mg-ATP, 0.3 Na2-GTP, 5 QX314 (in mM) (pH adjusted to 7.3 with NMDG).

544 In the whole-cell patch experiments, cells with a holding current lower than -100 pA or a change in the input resistance more than $30 \%$ were rejected.

\section{$\mathrm{Ca}^{2+}$ and NE imaging in brain slices}

AAV-GFAP104-jRCaMP1a or AAV-GFAP104-GRABNE2m virus was injected, and coronal brain slices were prepared as described above. Imaging was acquired at 0.5 to 1 frame per second with a $60 \mathrm{X}$ waterimmersion objective lens, and a 585-nm fluorescent imaging filter or 488-nm fluorescent imaging filter was utilized for jRCaMP1a or GRABNE2m imaging, respectively. Fluorescence Imaging was acquired with Imaging Workbench (Indec Biosystems), and analyzed with ImageJ software (NIH).

\section{Hippocampal fEPSP recording}

555 Hippocampal slices were transferred to a submerged recording chamber and perfused with aCSF 556 flowing at $2 \mathrm{~mL} / \mathrm{min}$. Schaffer collateral was stimulated using a concentric bipolar electrode (CBBPE75, 557 FHC, Bowdoin, ME, USA), and fEPSP was recorded from stratum radiatum of CA1 using a glass pipette 558 filled with aCSF (1-3 M $\Omega$ ). Evoked fEPSP responses were amplified by an AC differential amplifier (DAM 
80, World Precision Instruments, FL, USA) and digitized by BNC2110 (National Instruments). The slope of fEPSP response was analyzed by WinLTP v2.01 software (WinLTP Ltd., The University of Bristol, UK). The stimulation intensity was adjusted to obtain fEPSP slopes of $40-50 \%$ to the maximum. During recordings, bath temperature was maintained at $28 \pm 1^{\circ} \mathrm{C}$ by temperature controller (TC344B, Warner Instrument Corporation). Basal fEPSP response was monitored by electrical stimulations at $0.067 \mathrm{~Hz}$, and various stimulation protocols were delivered to test synaptic plasticity. High-frequency stimulation (HFS) consisted of 100 stimuli delivered at $100 \mathrm{~Hz} .10 \mathrm{~Hz}$ stimulation consisted of 900 stimuli delivered at 10 $\mathrm{Hz}$. Low-frequency stimulation (LFS) (1 Hz, 900 stimulations) consisted of 900 stimuli delivered at $1 \mathrm{~Hz}$. electrodes, borosilicate theta glass were fabricated and filled with aCSF to deliver focal stimulation on two independent pathways. Stimulation intensity was adjusted to acquire two independent pathways during paired-pulse ratio (PPR) test with $50 \mathrm{~ms}$ intervals, and the amplitude of each fEPSP was $0.1-0.4 \mathrm{mV}$.

\section{Primary astrocyte culture preparation}

573 Primary astrocytes were prepared from P0-P3 of C57BL/6 mouse as described (Lee et al., 2007).

574 Briefly, forebrain of mouse pup was dissected free of adherent meninges, minced and dissociated into 575 single cell suspension by trituration. Cells were grown in Dulbecco's modified Eagle's medium (DMEM, 576 Invitrogen) supplemented with $25 \mathrm{mM}$ glucose, $10 \%$ heat-inactivated horse serum, $10 \%$ heat-inactivated 577 fetal bovine serum, $2 \mathrm{mM}$ glutamine and 1,000 units/ml penicillin-streptomycin. Cultured astrocytes were 578 maintained at $37^{\circ} \mathrm{C}$ in a humidified $5 \% \mathrm{CO}_{2}$ incubator. On the third day of culture (postnatal days 3, PND 5793 ), cells were vigorously washed with repeated pipetting and the media was replaced to get rid of debris 580 and other floating cell types.

581 The day after wash (PND 4), cells were re-plated onto cover-glass coated with $0.1 \mathrm{mg} / \mathrm{ml}$ Poly D-Lysine 582 (PDL), while various shRNAs were delivered to astrocytes by by electroporation. The electroporation was performed using the Microporator (Invitrogen) with an optimized voltage protocol (1200V, 2 pulses, $20 \mathrm{~ms}$ pulse width). Cell number for each transfection was around $2 \times 10^{6}$. The following constructs were used; 5 mg pSicoR-SR-shRNA for SR knockdown experiment, $5 \mathrm{mg}$ pSicoR-Best1-shRNA for Best1 knockdown experiment, and additional $5 \mathrm{mg}$ pIRES2-mBest1-dsRED2 (shRNA insensitive form) or additional $5 \mathrm{mg}$ pIRES2-mBest1-dsRED2 (W93C mutant) for control experiments.

\section{Sensor cell preparation}

590 HEK293T cells were purchased from the Korean Cell Line Bank (Seoul National University) and cultured 591 in DMEM (Invitrogen) supplemented with 10\% fetal bovine serum (Invitrogen), $100 \mathrm{units} / \mathrm{ml}$ penicillin 592 (Invitrogen), and $100 \mathrm{mg} / \mathrm{ml}$ streptomycin (Invitrogen) at $37^{\circ} \mathrm{C}$ in a humidified $5 \% \mathrm{CO}_{2}$ incubator. One day 593 before the experiment for sniffer patch, HEK293T cells were transfected with 1:10 ratio of green 594 fluorescence protein (pEGFP-N1) and pCINeo-NR1-1/NR2A(2D-S1) (5 mg per $60 \mathrm{~mm}$ dish) or 1:3 red 
fluorescence protein (pDsRed) and pCINeo-NR1-1/NR2A(2D-S1) using Effectene (Qiagen) following the manufacturer's instructions. Additional $5 \mathrm{mM} \mathrm{APV}$ was supplemented in the medium to block the NMDA receptor-mediated cytotoxicity.

\section{Sniffer patch experiment}

600 Sniffer patch was composed of Fura-2 imaging for $\mathrm{Ca}^{2+}$ from astrocytes and current recording from 601 HEK293T cells expressing NR1-1/NR2A(2D-S1). On the day of experiment, the cover-slip in which 602 astrocytes and sensor cells were seeded was incubated with 5 mM Fura-2 AM (mixed with $5 \mathrm{ml}$ of $20 \%$ 603 Pluronic acid) (Invitrogen) for $40 \mathrm{~min}$, washed at room temperature, and subsequently transferred to the 604 microscope stage for imaging. External solution contained (in mM): $150 \mathrm{NaCl}, 10 \mathrm{HEPES}, 3 \mathrm{KCl}, 2 \mathrm{CaCl}_{2}$, $6052 \mathrm{MgCl}_{2}, 10$ glucose and ( $\mathrm{pH}$ adjusted to $\mathrm{pH} 7.3$ and osmolarity adjusted to $325 \mathrm{mOsmol} / \mathrm{kg}$ ). Intensity 606 images of $510 \mathrm{~nm}$ wavelength were taken at $340 \mathrm{~nm}$ and $380 \mathrm{~nm}$ excitation wavelengths by using iXon 607 EMCCD (ANDOR). To induce astrocytic $\mathrm{Ca}^{2+}, 500 \mu \mathrm{M}$ TFLLR was applied with pressure (20 lbf in-2, 608 100ms) using Picospritzer (Parker Instrument, USA). Two resulting images were used for 340/380 ratio 609 calculation in Imaging Workbench version 6.2 (Indec Systems). NR1-1A/NR2A(2D-S1)-mediated currents were recorded from HEK293T cells under voltage clamp $\left(V_{h}=-70 m V\right)$ using Axopatch 200A amplifier acquired with pClamp 10.4 (Molecular Devices). Recording electrodes (4-7M $\Omega$ ) were filled with $(\mathrm{mM})$ : $110 \mathrm{Cs}$-Gluconate, $30 \mathrm{CsCl}, 0.5 \mathrm{CaCl}_{2}, 10 \mathrm{HEPES}, 4 \mathrm{Mg}$-ATP, $0.3 \mathrm{Na}_{2}$-GTP, and 10 BAPTA (pH adjusted to 7.3 with $\mathrm{CsOH}$ and osmolarity adjusted to $290-310 \mathrm{mOsm} / \mathrm{kg}$ with sucrose). For simultaneous recording, Imaging Workbench was synchronized with pClamp 10.4.

\section{Two-cell assay}

617 To investigate co-release of D-serine and glutamate via Best1, two-cell assay was performed.

618 Preparation of sensor cell was performed as described above. For source cell preparation, HEK cells 619 were transfected with pIRES2-mBest1-dsRED2 or pIRES2-mBest1(W93C)-dsRED2 (7 mg per $60 \mathrm{~mm}$

620 dish) using Effectene (Qiagen). The internal solutions for source cell contained (in $\mathrm{mM}$ ); glutamate without 621 D-serine, $90 \mathrm{CsCl}, 50$ glutamate, 10 HEPES, $5\left(\mathrm{Ca}^{2+}\right)$-EGTA-NMDG, 2 MgCl , 0.3 Na2-GTP, 4 Mg-ATP $622(\mathrm{pH} 7.3$ with $\mathrm{CsOH}, 289 \mathrm{mOsmol}$ by adding sucrose); glutamate with D-serine, $40 \mathrm{CsCl}, 50$ glutamate, 50 623 D-serine, 10 HEPES, 5 ( $\mathrm{Ca}^{2+}$ )-EGTA-NMDG, $2 \mathrm{MgCl}_{2}$, 0.3 Na2-GTP, 4 Mg-ATP (pH 7.3 with CsOH, 289

$624 \mathrm{mOsmol}$ by adding sucrose). A pair of one sensor cell and one source cell were patched, and the 625 responsive current from sensor cells was measured under voltage clamp $\left(V_{h}=-70 \mathrm{mV}\right)$, while the source 626 cell is ruptured. Sensor current was measured as described above.

\section{Permeability assay}

629 To estimate the D-serine permeability of Best1, Best1 current was measured from Best1-expressing 630 HEK293T cell as previously performed (Lee et al., 2010), with various concentration of substitution for 
631 chloride to D-serine. The internal solution contained (in $\mathrm{mM}$ ); $100 \mathrm{CsCl}, 20$ tetraethylammonium (TEA)-Cl, $6328.7 \mathrm{CaCl}_{2}, 10 \mathrm{HEPES}, 10 \mathrm{BAPTA}, 3 \mathrm{Mg}$-ATP , $0.2 \mathrm{Na} 2$-GTP, and $0.5 \mathrm{MgCl}_{2}$, (pH was adjusted to 7.2 with $633 \mathrm{CsOH}$ ); when D-serine was included, it replaced an equimolar amount of $\mathrm{CsCl}$. Osmolarity was adjusted 634 to 287 mOsmol by adding sucrose. For these experiments, the external solution contained (in mM); 126

$635 \mathrm{NaCl}, 10 \mathrm{HEPES}, 20$ glucose, $1.8 \mathrm{CaCl}_{2}, 1.2 \mathrm{MgCl}_{2}$, and $10 \mathrm{TEA}-\mathrm{Cl}(\mathrm{pH} 7.4$ with $\mathrm{NaOH})$.

\section{Immunohistochemistry}

Adult mice were deeply anesthetized with $2 \%$ avertin and perfused with $0.1 \mathrm{M}$ PBS followed by $4 \%$ paraformaldehyde. Brains were postfixed in $4 \%$ paraformaldehyde at $4{ }^{\circ} \mathrm{C}$ for 24 hours and $30 \%$ sucrose at $4^{\circ} \mathrm{C}$ for 48 hours. Frozen brains were then cut into $30 \mu \mathrm{m}$ coronal cryosections. Sections were blocked in $0.1 \mathrm{M}$ PBS containing $0.3 \%$ Triton X-100 (Sigma) and 2\% Donkey Serum (GeneTex) for $30 \mathrm{~min}$ at room temperature. Primary antibody was applied at the appropriate dilution and incubated overnight at $4^{\circ} \mathrm{C}$.

643 Incubated sections were washed three times with 0.1 M PBS and incubated in secondary antibody for two 644 hours. After three rinses in 0.1 M PBS and DAPI staining at 1:1000 (Pierce), the sections were mounted 645 on polysine microscopic glass slides (Thermo Scientific). Images were scanned with the Axio Scan Z1 automated slide scanner (Zeiss) using ZEN 2 (blue edition) slidescan software (Zeiss).

\section{Western blotting}

649 Western blotting was performed as previous (Woo et al., 2012). Briefly, to test the expression of

650 NMDAR1, hippocampi were dissected from WT and Best1 KO, and the tissues were lysed with RIPA

651 buffer (50 mM Tris- $\mathrm{HCl}$, pH 7.4, $150 \mathrm{mM} \mathrm{NaCl}, 5 \mathrm{mM}$ EDTA, $1 \mathrm{mM} \mathrm{PMSF}$, and 1\% NP-40) containing a

652 protease-inhibitor cocktail. Obtained protein lysates were separated by SDS-PAGE using $10 \%$ gels and

653 blotted onto PVDF membranes. The blots were incubated with rabbit anti-NMDAR1 (ab109182, Abcam)

654 and anti- $\beta$-actin antibody (1:2,000; ab133626, Abcam). To test knockdown efficiency of SR, SR shRNA

655 was transfected as described above, and protein lysates were acquired three days after transfection. The 656 blots were incubated with mouse anti-SR (sc-365217, Santa Cruz) and anti- $\beta$-actin antibody. Appropriate 657 horseradish peroxidase-conjugated secondary antibodies (Jackson ImmunoResearch) were used for 658 detection by enhanced chemiluminescence (GE Healthcare). The band intensity was acquired by 659 ImageQuant LAS 4000 (GE Healthcare) and quantified using ImageJ software (NIH).

\section{Behavioral tests}

\section{Contextual fear conditioning}

663 Contextual fear conditioning test was performed as previously described (Jung et al., 2016). The 664 chamber with a stainless-steel floor was located in a sound-proof box with a camera mounted on its 665 ceiling (Med associates, Inc., St. Albans, VT, USA). On the first day, mice were allowed to explore the 666 chamber freely for $3 \mathrm{~min}$, and received six foot shocks separated by $1 \mathrm{~min}$ (0.4 mA, 2 seconds). Twenty- 
four hours after the training, the mice were placed in the chamber for $12 \mathrm{~min}$ and their behavioral responses were videotaped. Freezing response, defined as an absence of any movement except breathing for $>1 \mathrm{~s}$, was measured and divided by total time to.

\section{Passive avoidance test}

672 Passive avoidance test were performed as previously described (Jo et al., 2014). Briefly, mouse was

673 placed in the two-chamber (light and dark) box with a constant current shock generator (MED

674 Associates). On the acquisition, mouse was released into light chamber for one minute (habituation), and

675 a gate between two chambers was opened. When mouse passed the gate, the gate immediately is

676 closed, and aversive electric shock ( $0.5 \mathrm{~mA}, 2$ seconds) was delivered through the floor. After the delivery

677 of electric shock, mouse was returned to the home cage. Retention test was performed 24 hours after

678 acquisition test. For retention test, mouse was placed in the light chamber and the door opened 1 minute

679 later, and the latency of entry to dark chamber was recorded.

\section{Morris water maze}

682 Morris water maze experiments were performed as previously described (Park et al., 2015). Briefly, for

683 hidden platform Morris water maze experiments, animals were trained to find a hidden platform $(10 \mathrm{~cm}$

684 diameter, $1 \mathrm{~cm}$ under the water surface) placed in a fixed location in a water maze (1.2 $\mathrm{m}$ diameter) filled with water $\left(25^{\circ} \mathrm{C}\right)$ made opaque by the addition of nontoxic white paint (Weather tough Forte, Bristol Paints). The water maze was surrounded by a black circular curtain (placed $70 \mathrm{~cm}$ away) that held 3 salient visual cues. The releasing point was randomly distributed across 4 quadrants of the pool and the animal was allowed maximum $60 \mathrm{sec}$ to find the hidden platform. If escape did not occur within $60 \mathrm{sec}$, the animal was manually guided to the platform where they stayed on for $30 \mathrm{sec}$. The training consisted of 4 trials/day (10 min inter-trial interval, ITI) for 7 days. On training days 4 and 8, animals were given 60 sec probe tests to test their spatial memory. After 7 days of acquisition, the hidden platform was placed on the opposite quadrant to test spatial reversal learning for 3 additional days and the final probe test. For the D-serine application, D-serine $(600 \mathrm{mg} / \mathrm{kg}$ ) was injected intraperitoneally 20 min before the first trial of each day during acquisition session. Control group consisted of half saline-injected mice and half naive mice. The amount of saline injection was set equal to the amount of saline in which D-serine was dissolved. Neither D-serine nor saline was injected during the following reversal learning session.

697 For the visible platform test, animals were trained to find a visible platform (10 cm diameter, $1 \mathrm{~cm}$ above the water surface) marked with a salient black tape for 2 days (4 trials/day, 10 min ITI). If the animal found

699 the platform, the animal remained on the platform for $30 \mathrm{sec}$. During the test session after acquisition (day

7003 , trial 9), the platform was moved to a new location (adjacent right quadrant). And the animals were

701 released in the pool equidistant from the original and new location. An automated tracking system

702 (Noldus, Netherlands) was used to monitor and analyze the number of platform crossing, and the amount 703 of time spent in each of the four quadrants. 
704 For the Best1 rescue experiments, experimental conditions were set as above, except the size of water

705 maze (1.5 m diameter) and the number of trials ( 3 trials/day, $10 \mathrm{~min} \mathrm{ITI).}$

706

\section{Statistical analysis}

708 Statistical analyses were performed using Prism 9. All data were presented as mean \pm SEM. No

709 statistical method was used to predetermine sample size. Sample sizes were empirically determined

710 based on our previous experiences or other literatures. Experimental groups were balanced in terms of

711 animal age, sex and weight. Animals were genotyped prior to the experiment, and they were treated in

712 the same way. Animals were randomly and evenly allocated to each experimental condition. To perform

713 the group allocation in a blind manner during data collection, animal preparations and experiments were

714 carried out by different researchers. Statistical significance was set as ${ }^{*} p<0.05,{ }^{* *} p<0.01,{ }^{* * *} p<0.001$. 


\section{Supplemental Information}

A

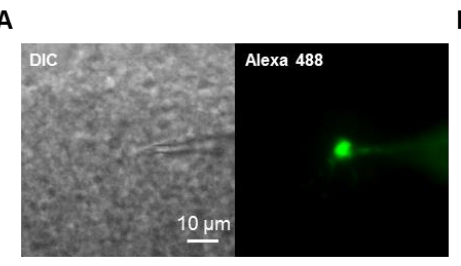

B

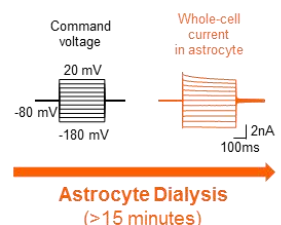

D

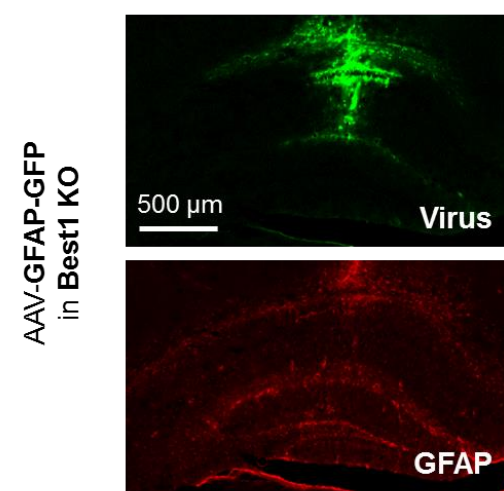

E hippocampal CA1 with astrocyte-specific AAV viruses. Related to Figures 1, 3, 7 cells (green) show Best1 expression (magenta).

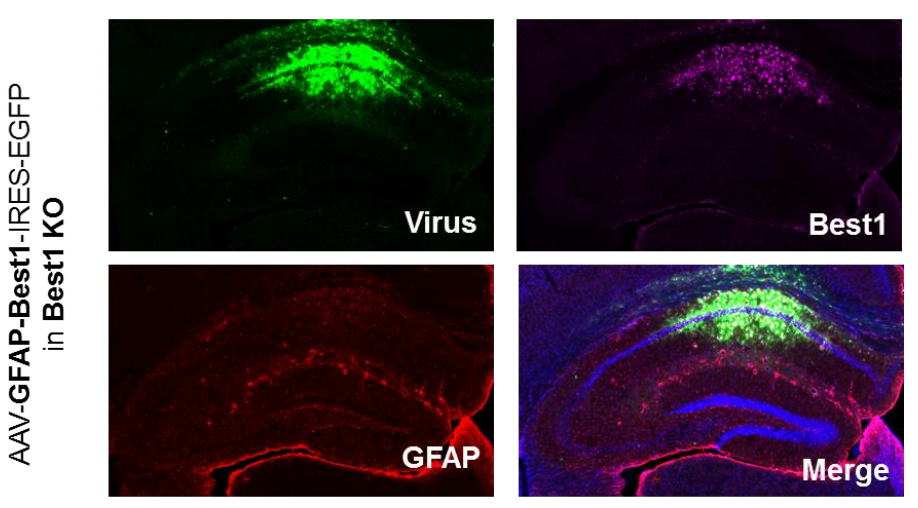

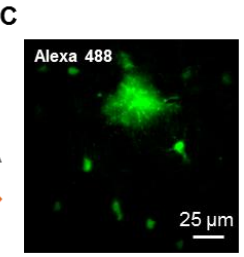
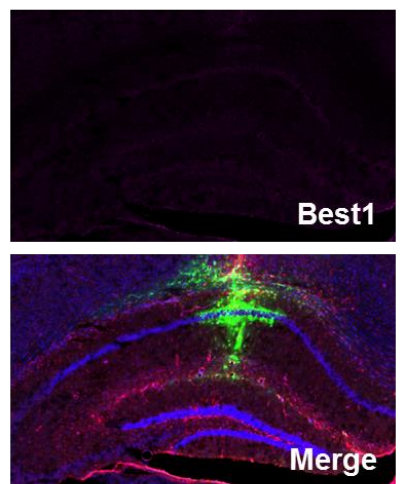

\section{Figure S1. Astrocytic dialysis through patch-clamp recording and rescue of astrocytic Best1 in}

(A) DIC and fluorescence image of hippocampal astrocyte in CA1 stratum radiatum after whole-cell patch recording with BAPTA, Alexa 488 fluorophore-containing internal patch pipette. (B) Passive conductance from astrocytes during astrocytic dialysis for more than 15 minutes. $(C)$ Representative images of astrocytic dialysis with Alexa 488 fluorophore. (D) AAV-GFAP-GFP (control virus) injection into hippocampal CA1 of Best1 KO. Virus-infected cells (green) show no Best1 expression (magenta). (E) AAV-GFAP-Best1-IRES-EGFP (rescue virus) injection into hippocampal CA1 of Best1 KO. Virus-infected 
A

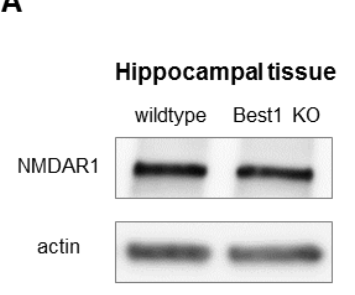

B

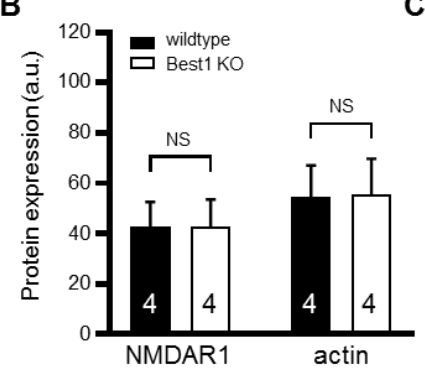

C

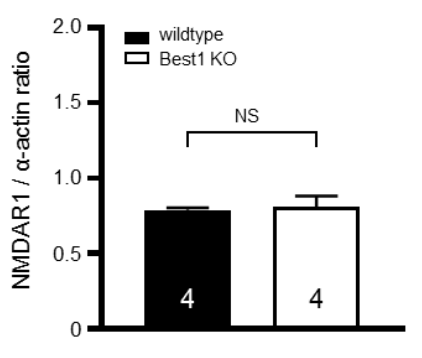

727 Figure S2. Hippocampal NMDAR1 expression in WT and Best1 KO. Related to Figures 1

728 (A) Western blot results for NMDAR1 and actin from hippocampal tissues of WT and Best1 KO. (B)

729 Quantification of the western blot results from hippocampal tissues of WT and Best1 KO. Number

730 indicates animal number of each condition. (C) Ratio of NMDAR1 / actin expression in hippocampi of

731 wildtype and Best1 KO. Number indicates animal number of each condition. Data are presented as mean

$732 \pm$ SEM. NS $>0.05$. 


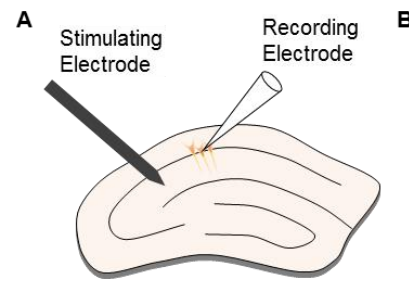

D

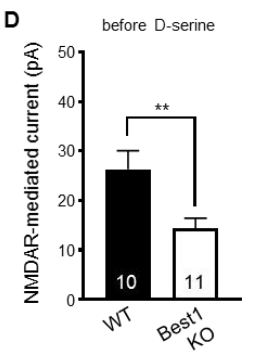

H
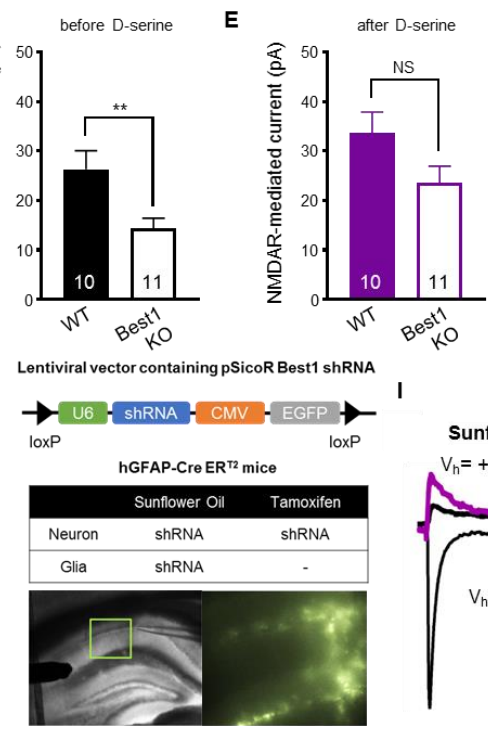

K

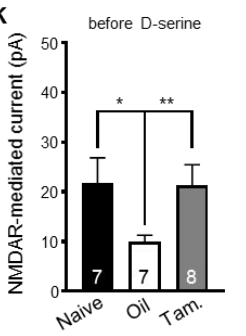

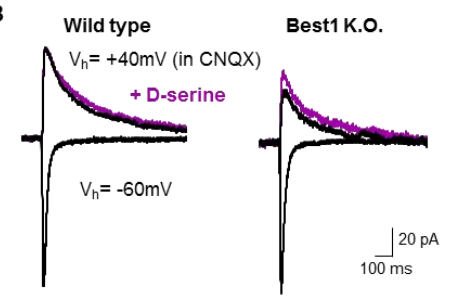

F

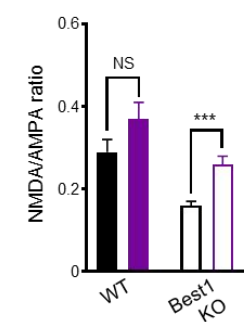

$\circ$
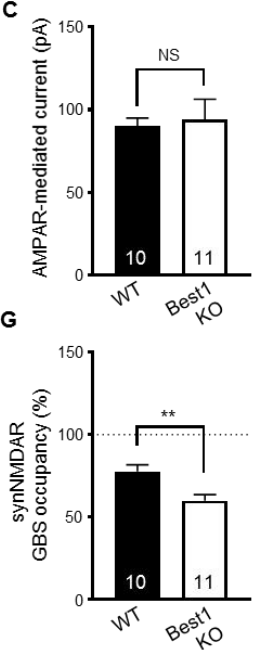

$\mathbf{J}$

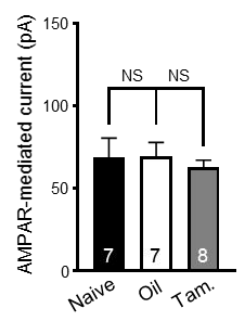

M

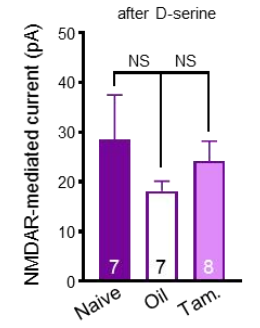

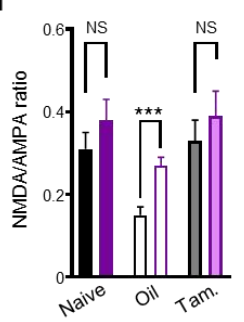

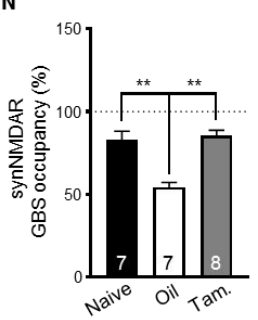

Figure S3. SynNMDAR in WT and Best1 KO. Related to Figures 1

(A) Scheme of synNMDAR recording. (B) Representative AMPAR-, NMDAR- mediated current, and NMDAR-mediated current with $100 \mu \mathrm{M}$ D-serine application in WT and Best1 KO. (C-G) Summary graph of AMPAR (C), NMDAR (D), NMDAR after $100 \mu \mathrm{M}$ D-serine application (E), NMDAR/AMPAR ratio (F), and estimated synNMDAR glycine modulatory site (GMS) occupancy $(\%)(G)$ in WT and Best1 KO. $(H)$ Cell-type specific manipulation of Best1 shRNA in hGFAP-CreERT2 mice. General knockdown of Best1 with sunflower oil (Oil, control), and astrocyte-specific rescue of Best1 with tamoxifen (Tam.) application. (I) Representative AMPAR-, NMDAR- mediated current, and NMDAR-mediated current with $100 \mu \mathrm{M}$ serine application from Oil- and Tam.-injected group. (J-I) Summary graph of AMPAR (J), NMDAR (K), NMDAR after $100 \mu \mathrm{M}$ D-serine application (L), NMDAR/AMPAR ratio (M), and estimated synaptic NMDAR glycine modulatory site (GMS) occupancy (\%) (N) in naïve, Oil- and Tam.-injected group. Data are presented as mean \pm SEM. ${ }^{*} p<0.05 ;{ }^{* *} p<0.01 ;{ }^{* * *} p<0.001$. 
A

\begin{tabular}{|c|c|c|c|c|}
\hline \multicolumn{2}{|c|}{$\begin{array}{l}\text { Co-expression in } \\
\text { HEK293T cells }\end{array}$} & $\begin{array}{c}\text { SR-EGFP } \\
+ \\
\mathrm{mCh}\end{array}$ & \multicolumn{2}{|c|}{$\begin{array}{c}\text { SR-EGFP } \\
+ \\
\text { SR shRNA-mCh }\end{array}$} \\
\hline \multirow{2}{*}{$\begin{array}{l}\text { Knockdown } \\
\text { Test }\end{array}$} & \multicolumn{2}{|c|}{\begin{tabular}{c|} 
Control \\
\end{tabular}} & \multicolumn{2}{|c|}{ shRNA } \\
\hline & $\begin{array}{c}\mathrm{mCh} \text { (RG, } \\
\text { reference gene) }\end{array}$ & $\begin{array}{c}\text { EGFP (TG, target } \\
\text { gene) }\end{array}$ & $\begin{array}{c}\mathrm{mCh} \text { (RG, } \\
\text { reference gene) }\end{array}$ & $\begin{array}{c}\text { EGFP (TG, target } \\
\text { gene) }\end{array}$ \\
\hline \multirow{3}{*}{ triplet } & 14.33 & \begin{tabular}{|l|}
13.27 \\
\end{tabular} & 13.94 & 14.55 \\
\hline & 14.36 & 13.45 & 13.82 & 14.43 \\
\hline & 14.38 & 13.43 & 13.84 & 14.48 \\
\hline average & 14.36 & 13.38 & 13.87 & 14.49 \\
\hline$\Delta \mathrm{Ct}$ (RG - TG) & \multicolumn{2}{|c|}{0.97} & \multicolumn{2}{|c|}{-0.62} \\
\hline $\begin{array}{c}\Delta \Delta \mathrm{Ct} \text { (sample - } \\
\text { control) }\end{array}$ & \multicolumn{2}{|c|}{0.00} & \multicolumn{2}{|c|}{-1.59} \\
\hline \% expression & \multicolumn{2}{|c|}{100.00} & \multicolumn{2}{|c|}{33.14} \\
\hline knockdown \% & \multicolumn{2}{|c|}{0.00} & \multicolumn{2}{|c|}{66.86} \\
\hline
\end{tabular}

C

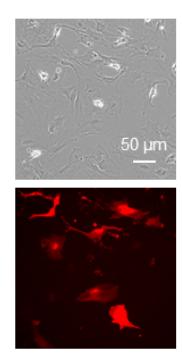

D

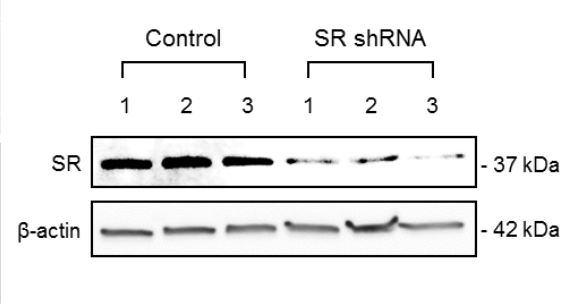

B

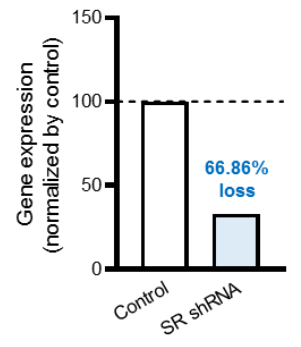

E

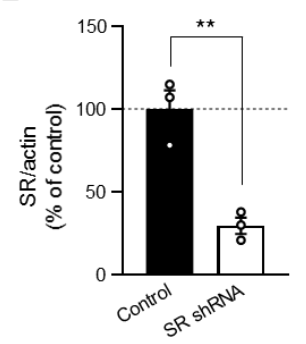

Figure S4. Validation of SR shRNA. Related to Figures 2 and 3

748 (A) Upper: SR shRNA efficiency was accessed by co-expression of SR-EGFP and shRNA (or control

749 vector) in HEK293T cells. Lower: Results of Quantitative RT-PCR and analyzed results by $\Delta \Delta$ Ct method.

750 (B) Summarized results of mRNA knockdown efficiency of mSR shRNA. (C) Transfection of SR shRNA in 751 primary astrocytes. (D) Western blot results for SR in control and SR shRNA conditions. Numbers

752 indicate different culture batches. (E) Ratio of SR / actin expression. Dots indicate different culture

753 batches. Data are presented as mean \pm SEM. ${ }^{*} p<0.05 ;{ }^{* *} p<0.01$; ${ }^{* * *} p<0.001$. 
A

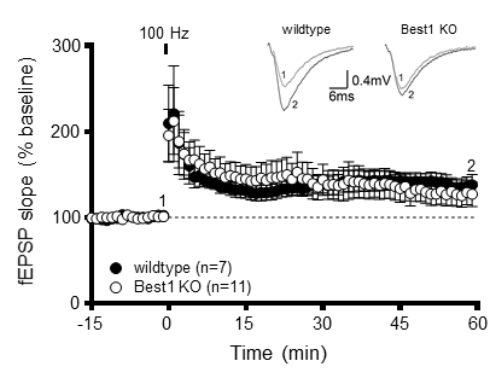

C

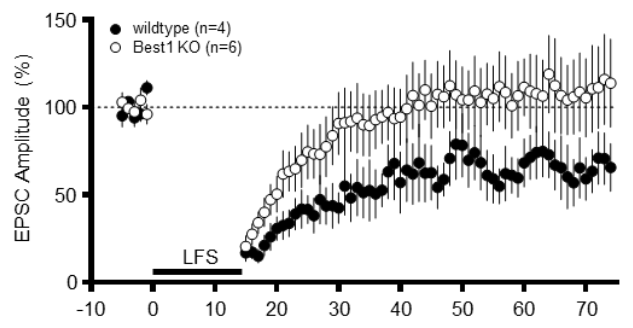

E

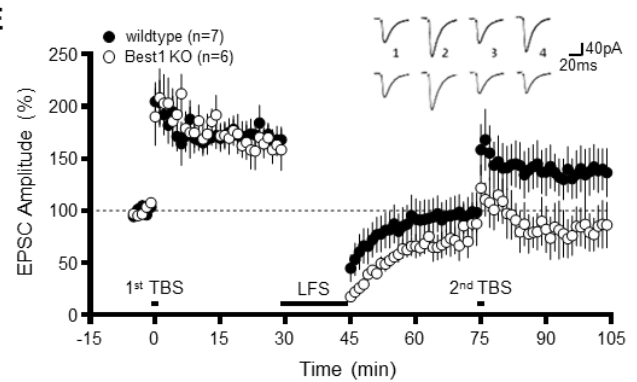

B

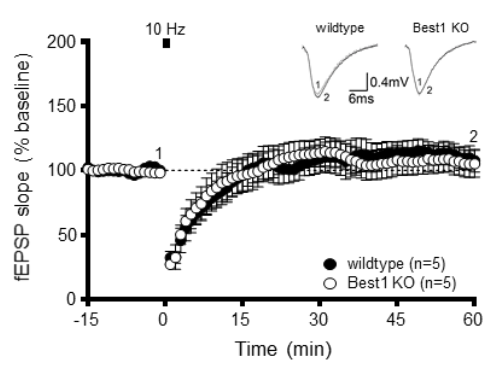

D

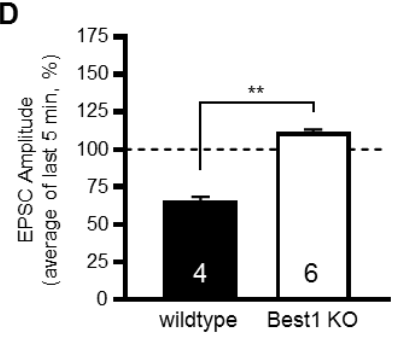

F

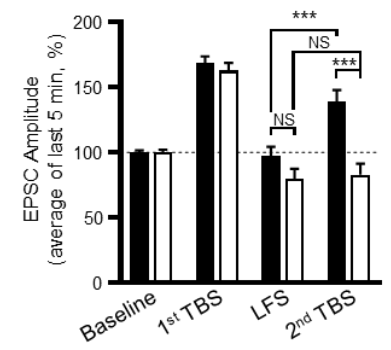

754

755

756

757

758

759

760

761

762

763

Figure S5. Hippocampal synaptic plasticity in Best1 KO. Related to Figures 3 and 6

(A) HFS (100 stimuli at $100 \mathrm{~Hz}$ )-induced LTP in WT and Best1 $\mathrm{KO}$ under fEPSP recording. (B) $10 \mathrm{~Hz}$ stimulation ( 900 stimuli ) in WT and Best1 KO under fEPSP recording. (C) LFS (900 stimuli at $1 \mathrm{~Hz}$ )induced LTD in WT and Best1 KO under excitatory postsynaptic current (EPSC) recording from CA1 pyramidal neuron with whole-cell patch-clamp. (D) Summary graph of LTD induction in WT and Best1 KO under EPSC recording. (E and F) Time course of the normalized EPSC changes (E) and summary graph (F) of $1^{\text {st }}$ TBS (theta-burst stimulation)-induced potentiation, LFS-induced depotentiation, and $2^{\text {nd }}$ TBSinduced repotentiation in WT and Best1 KO. Data are presented as mean \pm SEM. . ${ }^{*} p<0.05 ;{ }^{* *} p<0.01$; ${ }^{* * *} p<0.001$. 
A
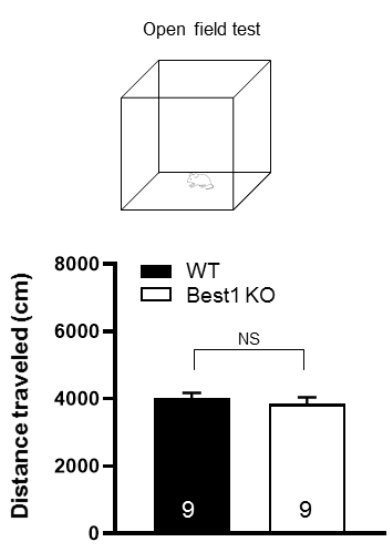

C

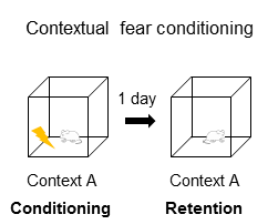

D
B
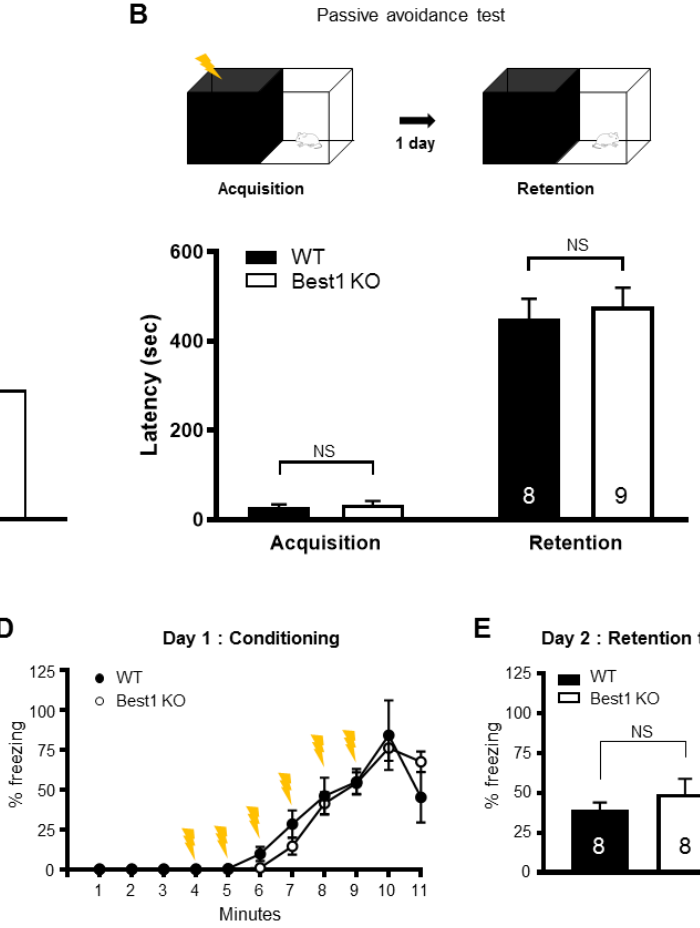

E Day 2 : Retention test

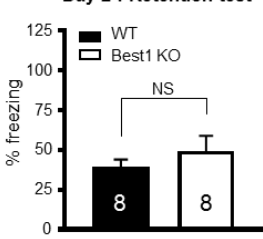

Figure S6. Best1 KO mice have impaired reversal learning, but not other hippocampus-dependent spatial learning. Related to Figure 7

(A) Top, Open field test (OFT). Bottom, Summary graph of distance traveled during OFT. (B) Top, Passive avoidance test (PAT). Bottom, Summary graph of latency to the dark room before and after shock-association in WT and Best1 KO. (C) Contextual fear conditioning (CFC). Retention test was performed in the same context, the day after fear conditioning with electric shock (yellow symbol). (D) Percentage of freezing time during conditioning session in WT (black) and Best1 KO (white). Yellow symbols represent the delivery of electric shock. (E) Percentage of freezing time in retention session the day after conditioning. Numbers indicate the number of animals. Data are presented as mean \pm SEM. ${ }^{*} \mathrm{p}$ $<0.05 ;{ }^{* *} p<0.01 ;{ }^{* * *} p<0.001$. 

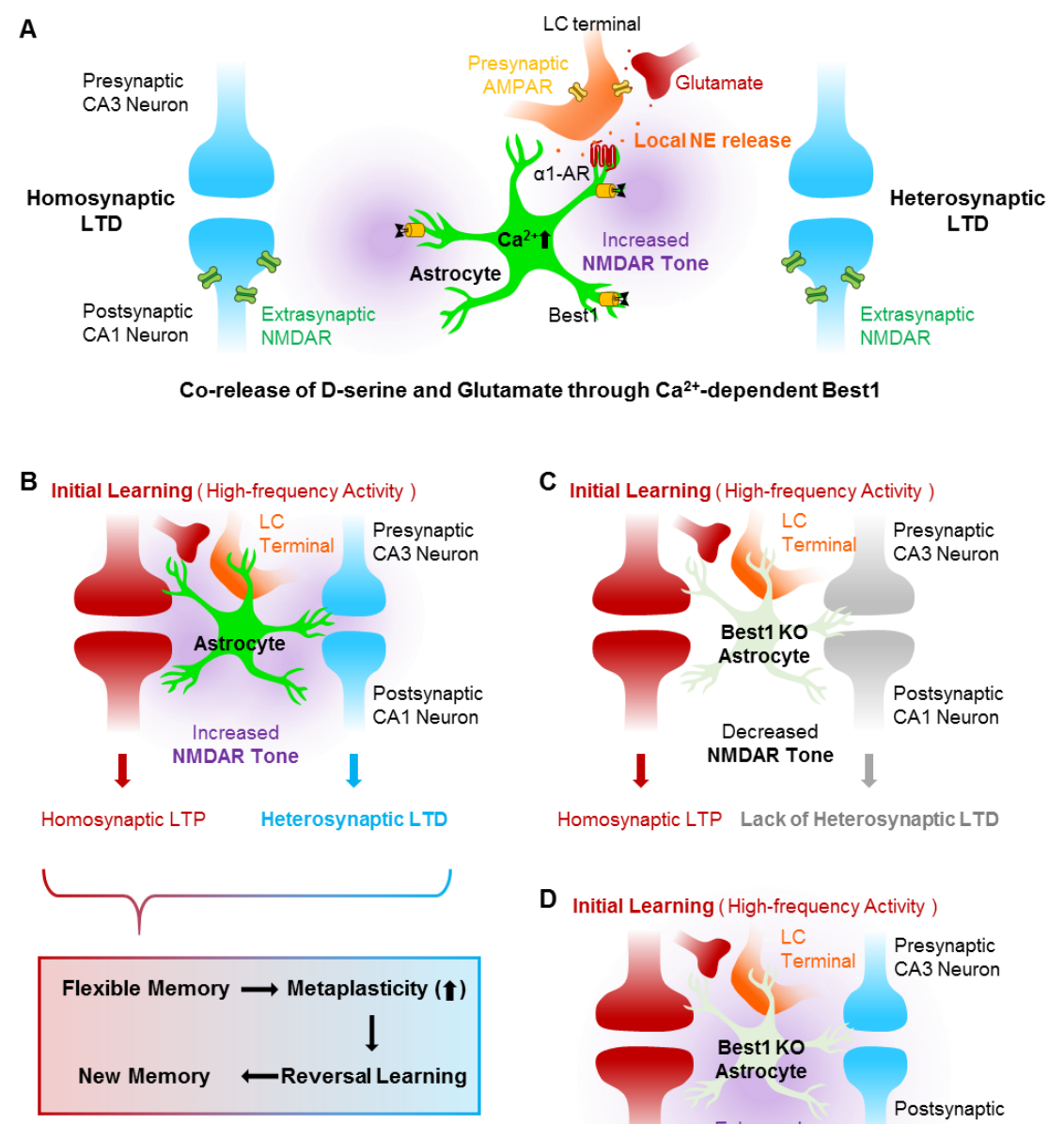

\section{Figure S7. Schematic illustration of a working model for Best1 in flexible memory. Related to}

777 Figure 7

778 (A) NMDAR tone is regulated by astrocytic co-release of D-serine and Glutamate through $\mathrm{Ca}^{2+}$-dependent

779 Best1. (B) Local NE release activates astrocytes, increases NMDAR tone, and induces heterosynaptic

780 LTD. Heterosynaptic LTD enable further plasticity (metaplasticity) and reversal learning. (C)

781 Heterosynaptic LTD is impaired in Best1 KO. (D) The impaired heterosynaptic LTD is restored by D-

782 serine application. 


\section{References}

Abraham, W.C., and Bear, M.F. (1996). Metaplasticity: the plasticity of synaptic plasticity. Trends in neurosciences 19, 126-130.

Araque, A., Li, N., Doyle, R.T., and Haydon, P.G. (2000). SNARE Protein-Dependent Glutamate Release from Astrocytes. The Journal of Neuroscience 20,666-673.

Atzori, M., Cuevas-Olguin, R., Esquivel-Rendon, E., Garcia-Oscos, F., Salgado-Delgado, R.C., Saderi, N., Miranda-Morales, M., Trevino, M., Pineda, J.C., and Salgado, H. (2016). Locus Ceruleus Norepinephrine Release: A Central Regulator of CNS Spatio-Temporal Activation? Frontiers in synaptic neuroscience 8 , 25. Boone, K.B., Ghaffarian, S., Lesser, I.M., Hill-Gutierrez, E., and G. Berman, N. (1993). Wisconsin card sorting test performance in healthy, older adults: Relationship to age, sex, education, and IQ. Journal of Clinical Psychology 49, 54-60.

Bushong, E.A., Martone, M.E., Jones, Y.Z., and Ellisman, M.H. (2002). Protoplasmic astrocytes in CA1 stratum radiatum occupy separate anatomical domains. The Journal of neuroscience : the official journal of the Society for Neuroscience 22, 183-192.

Chen, J., Tan, Z., Zeng, L., Zhang, X., He, Y., Gao, W., Wu, X., Li, Y., Bu, B., Wang, W., et al. (2013a). Heterosynaptic long-term depression mediated by ATP released from astrocytes. Glia 61, 178-191. Chen, J.Y., Lonjers, P., Lee, C., Chistiakova, M., Volgushev, M., and Bazhenov, M. (2013b). Heterosynaptic plasticity prevents runaway synaptic dynamics. The Journal of neuroscience : the official journal of the Society for Neuroscience 33, 15915-15929.

Chen, P.E., Geballe, M.T., Katz, E., Erreger, K., Livesey, M.R., O'Toole, K.K., Le, P., Lee, C.J., Snyder, J.P., Traynelis, S.F., et al. (2008). Modulation of glycine potency in rat recombinant NMDA receptors containing chimeric NR2A/2D subunits expressed in Xenopus laevis oocytes. The Journal of physiology 586, 227-245.

Craven, R.M., Priddle, T.H., Crow, T.J., and Esiri, M.M. (2005). The locus coeruleus in schizophrenia: a postmortem study of noradrenergic neurones. Neuropathology and applied neurobiology 31, 115-126. D'Cruz, A.M., Ragozzino, M.E., Mosconi, M.W., Shrestha, S., Cook, E.H., and Sweeney, J.A. (2013).

Reduced behavioral flexibility in autism spectrum disorders. Neuropsychology 27, 152-160.

De Pitta, M., Brunel, N., and Volterra, A. (2016). Astrocytes: Orchestrating synaptic plasticity? Neuroscience 323, 43-61. Dezfuli, G., Martin, L., Olson, T., and Kellar, K.J. (2019). The Decline of Glutamate-Stimulated Norepinephrine Release in Aging Rat Brain. The FASEB Journal 33, 501.511-501.511. Ding, F., O'Donnell, J., Thrane, A.S., Zeppenfeld, D., Kang, H., Xie, L., Wang, F., and Nedergaard, M. (2013). alpha1-Adrenergic receptors mediate coordinated Ca2+ signaling of cortical astrocytes in awake, behaving mice. Cell calcium 54, 387-394.

Do-Monte, F.H., Allensworth, M., and Carobrez, A.P. (2010). Impairment of contextual conditioned fear extinction after microinjection of alpha-1-adrenergic blocker prazosin into the medial prefrontal cortex. Behavioural brain research 211, 89-95.

Dong, Z., Bai, Y., Wu, X., Li, H., Gong, B., Howland, J.G., Huang, Y., He, W., Li, T., and Wang, Y.T. (2013). Hippocampal long-term depression mediates spatial reversal learning in the Morris water maze. Neuropharmacology 64, 65-73. Dudek, S.M., and Bear, M.F. (1993). Bidirectional long-term modification of synaptic effectiveness in the adult and immature hippocampus. The Journal of neuroscience : the official journal of the Society for Neuroscience 13, 2910-2918. 
Duffy, S., Labrie, V., and Roder, J.C. (2008). D-serine augments NMDA-NR2B receptor-dependent hippocampal long-term depression and spatial reversal learning. Neuropsychopharmacology : official publication of the American College of Neuropsychopharmacology 33, 1004-1018. Duffy, S., and MacVicar, B.A. (1995). Adrenergic calcium signaling in astrocyte networks within the hippocampal slice. The Journal of neuroscience : the official journal of the Society for Neuroscience 15, 5535-5550.

Dyer-Reaves, K., Goodman, A.M., Nelson, A.R., and McMahon, L.L. (2019). Alpha1-Adrenergic Receptor Mediated Long-Term Depression at CA3-CA1 Synapses Can Be Induced via Accumulation of Endogenous Norepinephrine and Is Preserved Following Noradrenergic Denervation. Frontiers in synaptic neuroscience 11, 27. Egawa, K., and Fukuda, A. (2013). Pathophysiological power of improper tonic GABA(A) conductances in mature and immature models. Frontiers in neural circuits 7, 170.

Etienne, V., Marin-Lamellet, C., and Laurent, B. (2013). Mental flexibility impairment in drivers with early Alzheimer's disease: A simulator-based study. IATSS Research 37, 16-20.

Feng, J., Zhang, C., Lischinsky, J.E., Jing, M., Zhou, J., Wang, H., Zhang, Y., Dong, A., Wu, Z., Wu, H., et al. (2019). A Genetically Encoded Fluorescent Sensor for Rapid and Specific In Vivo Detection of Norepinephrine. Neuron 102, 745-761 e748.

Gandal, M.J., Anderson, R.L., Billingslea, E.N., Carlson, G.C., Roberts, T.P., and Siegel, S.J. (2012). Mice with reduced NMDA receptor expression: more consistent with autism than schizophrenia? Genes, brain, and behavior 11, 740-750.

Ghersi, C., Bonfanti, A., Manzari, B., Feligioni, M., Raiteri, M., and Pittaluga, A. (2003). Pharmacological heterogeneity of release-regulating presynaptic AMPA/kainate receptors in the rat brain: study with receptor antagonists. Neurochemistry international 42, 283-292.

Guarino, A., Favieri, F., Boncompagni, I., Agostini, F., Cantone, M., and Casagrande, M. (2018). Executive Functions in Alzheimer Disease: A Systematic Review. Frontiers in aging neuroscience 10, 437. Han, Y.E., Kwon, J., Won, J., An, H., Jang, M.W., Woo, J., Lee, J.S., Park, M.G., Yoon, B.E., Lee, S.E., et al. (2019). Tweety-homolog (Ttyh) Family Encodes the Pore-forming Subunits of the Swelling-dependent Volume-regulated Anion Channel (VRACswell) in the Brain. Exp Neurobiol 28, 183-215.

Henneberger, C., Papouin, T., Oliet, S.H., and Rusakov, D.A. (2010). Long-term potentiation depends on release of D-serine from astrocytes. Nature 463, 232-236.

Hertz, L., Lovatt, D., Goldman, S.A., and Nedergaard, M. (2010). Adrenoceptors in brain: cellular gene expression and effects on astrocytic metabolism and $[\mathrm{Ca}(2+)]$ i. Neurochemistry international $57,411-$ 420.

Holtzclaw, L.A., Pandhit, S., Bare, D.J., Mignery, G.A., and Russell, J.T. (2002). Astrocytes in adult rat brain express type 2 inositol 1,4,5-trisphosphate receptors. Glia 39, 69-84.

Homan, P., Lin, Q., Murrough, J.W., Soleimani, L., Bach, D.R., Clem, R.L., and Schiller, D. (2017). Prazosin during threat discrimination boosts memory of the safe stimulus. Learning \& memory 24, 597-601. Howells, F.M., and Russell, V.A. (2008). Glutamate-stimulated release of norepinephrine in hippocampal slices of animal models of attention-deficit/hyperactivity disorder (spontaneously hypertensive rat) and depression/anxiety-like behaviours (Wistar-Kyoto rat). Brain research 1200, 107-115. Huang, Y.J., Lin, C.H., Lane, H.Y., and Tsai, G.E. (2012). NMDA Neurotransmission Dysfunction in Behavioral and Psychological Symptoms of Alzheimer's Disease. Current neuropharmacology 10, $272-$ 285.

Hulme, S.R., Jones, O.D., Raymond, C.R., Sah, P., and Abraham, W.C. (2014). Mechanisms of heterosynaptic metaplasticity. Philosophical transactions of the Royal Society of London Series B, Biological sciences 369, 20130148. 
Izquierdo, A., Brigman, J.L., Radke, A.K., Rudebeck, P.H., and Holmes, A. (2017). The neural basis of reversal learning: An updated perspective. Neuroscience 345, 12-26. Jacobowitz, D.M. (1979). Hypothesis for the Local Control of Norepinephrine Release. 1792-1794. Khakh, B.S., and Sofroniew, M.V. (2015). Diversity of astrocyte functions and phenotypes in neural circuits. Nat Neurosci 18, 942-952.

878 Kim, J.I., Lee, H.R., Sim, S.E., Baek, J., Yu, N.K., Choi, J.H., Ko, H.G., Lee, Y.S., Park, S.W., Kwak, C., et al. (2011). PI3Kgamma is required for NMDA receptor-dependent long-term depression and behavioral flexibility. Nat Neurosci 14, 1447-1454. Kwak, H., Koh, W., Kim, S., Song, K., Shin, J.I., Lee, J.M., Lee, E.H., Bae, J.Y., Ha, G.E., Oh, J.E., et al. (2020). Astrocytes Control Sensory Acuity via Tonic Inhibition in the Thalamus. Neuron. Labrie, V., Duffy, S., Wang, W., Barger, S.W., Baker, G.B., and Roder, J.C. (2009). Genetic inactivation of D-amino acid oxidase enhances extinction and reversal learning in mice. Learning \& memory 16, 28-37. Laube, B., Kuhse, J., and Betz, H. (2000). Kinetic and mutational analysis of Zn2+ modulation of recombinant human inhibitory glycine receptors. The Journal of physiology 522 Pt 2, 215-230. Le Meur, K., Galante, M., Angulo, M.C., and Audinat, E. (2007). Tonic activation of NMDA receptors by ambient glutamate of non-synaptic origin in the rat hippocampus. The Journal of physiology 580, 373383. of synaptic NMDA receptors. The Journal of physiology 581, 1057-1081. Lee, G., and Zhou, Y. (2019). NMDAR Hypofunction Animal Models of Schizophrenia. Frontiers in molecular neuroscience 12, 185.

Li, J., Chai, A., Wang, L., Ma, Y., Wu, Z., Yu, H., Mei, L., Lu, L., Zhang, C., Yue, W., et al. (2015). Synaptic PRex1 signaling regulates hippocampal long-term depression and autism-like social behavior. Proceedings of the National Academy of Sciences of the United States of America 112, E6964-6972. Liu, D.D., Yang, Q., and Li, S.T. (2013). Activation of extrasynaptic NMDA receptors induces LTD in rat hippocampal CA1 neurons. Brain research bulletin 93, 10-16. Lu, W.-Y., Man, H.-Y., Ju, W., Trimble, W.S., MacDonald, J.F., and Wang, Y.T. (2001). Activation of Synaptic NMDA Receptors Induces Membrane Insertion of New AMPA Receptors and LTP in Cultured Hippocampal Neurons. Neuron 29, 243-254.

Lynch, G.S., Dunwiddie, T., and Gribkoff, V. (1977). Heterosynaptic depression: a postsynaptic correlate of long-term potentiation. Nature 266, 737-739.

Malva, J.O., Carvalho, A.P., and Carvalho, C.M. (1994). Modulation of dopamine and noradrenaline release and of intracellular $\mathrm{Ca} 2+$ concentration by presynaptic glutamate receptors in hippocampus. British journal of pharmacology 113, 1439-1447.

Marmorstein, L.Y., Wu, J., McLaughlin, P., Yocom, J., Karl, M.O., Neussert, R., Wimmers, S., Stanton, J.B., Gregg, R.G., Strauss, O., et al. (2006). The light peak of the electroretinogram is dependent on voltagegated calcium channels and antagonized by bestrophin (best-1). The Journal of general physiology 127 , 577-589.

Martchek, M., Thevarkunnel, S., Bauman, M., Blatt, G., and Kemper, T. (2006). Lack of evidence of neuropathology in the locus coeruleus in autism. Acta neuropathologica 111, 497-499.

Matchett, B.J., Grinberg, L.T., Theofilas, P., and Murray, M.E. (2021). The mechanistic link between selective vulnerability of the locus coeruleus and neurodegeneration in Alzheimer's disease. Acta neuropathologica.

Mather, M., Clewett, D., Sakaki, M., and Harley, C.W. (2016). Norepinephrine ignites local hotspots of neuronal excitation: How arousal amplifies selectivity in perception and memory. The Behavioral and brain sciences 39, e200. 
Morice, E., Billard, J.M., Denis, C., Mathieu, F., Betancur, C., Epelbaum, J., Giros, B., and NostenBertrand, M. (2007). Parallel loss of hippocampal LTD and cognitive flexibility in a genetic model of hyperdopaminergia. Neuropsychopharmacology : official publication of the American College of Neuropsychopharmacology 32, 2108-2116. Nam, M.H., Han, K.S., Lee, J., Won, W., Koh, W., Bae, J.Y., Woo, J., Kim, J., Kwong, E., Choi, T.Y., et al. (2019). Activation of Astrocytic mu-Opioid Receptor Causes Conditioned Place Preference. Cell reports 28, 1154-1166 e1155.

Navarrete, M., Cuartero, M.I., Palenzuela, R., Draffin, J.E., Konomi, A., Serra, I., Colie, S., CastanoCastano, S., Hasan, M.T., Nebreda, A.R., et al. (2019). Astrocytic p38alpha MAPK drives NMDA receptordependent long-term depression and modulates long-term memory. Nature communications 10, 2968. Nicholls, R.E., Alarcon, J.M., Malleret, G., Carroll, R.C., Grody, M., Vronskaya, S., and Kandel, E.R. (2008). Transgenic mice lacking NMDAR-dependent LTD exhibit deficits in behavioral flexibility. Neuron 58, 104117.

Oh, S.-J.J., and Lee, C.J. (2017). Distribution and Function of the Bestrophin-1 (Best1) Channel in the Brain. Experimental neurobiology 26, 113-121.

Oh, S.J., Han, K.S., Park, H., Woo, D.H., Kim, H.Y., Traynelis, S.F., and Lee, C.J. (2012). Protease activated receptor 1-induced glutamate release in cultured astrocytes is mediated by Bestrophin-1 channel but not by vesicular exocytosis. Mol Brain 5, 38.

Oh, S.J., Lee, J.M., Kim, H.B., Lee, J., Han, S., Bae, J.Y., Hong, G.S., Koh, W., Kwon, J., Hwang, E.S., et al. (2019). Ultrasonic Neuromodulation via Astrocytic TRPA1. Current biology : CB 29, 3386-3401 e3388. Okubo, Y., Kanemaru, K., Suzuki, J., Kobayashi, K., Hirose, K., and lino, M. (2018). Inositol 1,4,5trisphosphate receptor type 2 -independent $\mathrm{Ca} 2+$ release from the endoplasmic reticulum in astrocytes. Glia.

Ottestad-Hansen, S., Hu, Q.X., Follin-Arbelet, V.V., Bentea, E., Sato, H., Massie, A., Zhou, Y., and Danbolt, N.C. (2018). The cystine-glutamate exchanger (xCT, Slc7a11) is expressed in significant concentrations in a subpopulation of astrocytes in the mouse brain. Glia 66, 951-970.

Papouin, T., Henneberger, C., Rusakov, D.A., and Oliet, S. (2017). Astroglial versus Neuronal D-Serine: Fact Checking. Trends in neurosciences 40, 517-520.

Papouin, T., Ladepeche, L., Ruel, J., Sacchi, S., Labasque, M., Hanini, M., Groc, L., Pollegioni, L., Mothet, J.P., and Oliet, S.H. (2012). Synaptic and extrasynaptic NMDA receptors are gated by different endogenous coagonists. Cell 150, 633-646.

Park, H., Han, K.-S., Seo, J., Lee, J., Dravid, S.M., Woo, J., Chun, H., Cho, S., Bae, J., An, H., et al. (2015). Channel-mediated astrocytic glutamate modulates hippocampal synaptic plasticity by activating postsynaptic NMDA receptors. Molecular Brain 8, 1-16.

Park, H., Han, K.S., Oh, S.J., Jo, S., Woo, J., Yoon, B.E., and Lee, C.J. (2013). High glutamate permeability and distal localization of Best1 channel in CA1 hippocampal astrocyte. Molecular Brain 6.

Park, H., Oh, S.J., Han, K.S., Woo, D.H., Park, H., Mannaioni, G., Traynelis, S.F., and Lee, C.J. (2009). Bestrophin-1 encodes for the Ca2+-activated anion channel in hippocampal astrocytes. The Journal of neuroscience : the official journal of the Society for Neuroscience 29, 13063-13073.

Parpura, V., and Haydon, P.G. (2000). Physiological astrocytic calcium levels stimulate glutamate release to modulate adjacent neurons. Proceedings of the National Academy of Sciences of the United States of America 97, 8629-8634.

Parsons, M.P., and Raymond, L.A. (2014). Extrasynaptic NMDA receptor involvement in central nervous system disorders. Neuron 82, 279-293.

Petravicz, J., Fiacco, T.A., and McCarthy, K.D. (2008). Loss of IP3 Receptor-Dependent Ca2+ Increases in Hippocampal Astrocytes Does Not Affect Baseline CA1 Pyramidal Neuron Synaptic Activity. The Journal of Neuroscience 28, 4967-4973. 
Povysheva, N.V., and Johnson, J.W. (2012). Tonic NMDA receptor-mediated current in prefrontal cortical pyramidal cells and fast-spiking interneurons. Journal of neurophysiology 107, 2232-2243. Price, R., Mercuri, N.B., and Ledonne, A. (2021). Emerging Roles of Protease-Activated Receptors (PARs) in the Modulation of Synaptic Transmission and Plasticity. International journal of molecular sciences 22. Rorabaugh, J.M., Chalermpalanupap, T., Botz-Zapp, C.A., Fu, V.M., Lembeck, N.A., Cohen, R.M., and Weinshenker, D. (2017). Chemogenetic locus coeruleus activation restores reversal learning in a rat model of Alzheimer's disease. Brain : a journal of neurology 140, 3023-3038.

Rungta, R.L., Bernier, L.-P.P., Dissing-Olesen, L., Groten, C.J., LeDue, J.M., Ko, R., Drissler, S., and MacVicar, B.A. (2016). $\mathrm{Ca}(2+)$ transients in astrocyte fine processes occur via $\mathrm{Ca}(2+)$ influx in the adult mouse hippocampus. Glia 64, 2093-2103.

Rutten, K., Wallace, T.L., Works, M., Prickaerts, J., Blokland, A., Novak, T.J., Santarelli, L., and Misner, D.L. (2011). Enhanced long-term depression and impaired reversal learning in phosphodiesterase 4Bknockout (PDE4B-/-) mice. Neuropharmacology 61, 138-147.

Sah, P., Hestrin, S., and Nicoll, R.A. (1989). Tonic activation of NMDA receptors by ambient glutamate enhances excitability of neurons. Science $246,815-818$.

Sahlender, D.A., Savtchouk, I., and Volterra, A. (2014). What do we know about gliotransmitter release from astrocytes? Philosophical transactions of the Royal Society of London Series B, Biological sciences 369, 20130592.

Sason, H., Billard, J.M., Smith, G.P., Safory, H., Neame, S., Kaplan, E., Rosenberg, D., Zubedat, S., Foltyn, V.N., Christoffersen, C.T., et al. (2017). Asc-1 Transporter Regulation of Synaptic Activity via the Tonic Release of d-Serine in the Forebrain. Cerebral cortex 27, 1573-1587.

Scanziani, M., Nicoll, R.A., and Malenka, R.C. (1996). Heterosynaptic long-term depression in the hippocampus. Journal of physiology, Paris 90, 165-166.

Scheiderer, C.L., Dobrunz, L.E., and McMahon, L.L. (2004). Novel form of long-term synaptic depression in rat hippocampus induced by activation of alpha 1 adrenergic receptors. Journal of neurophysiology 91, 1071-1077.

Schwarz, L.A., and Luo, L. (2015). Organization of the locus coeruleus-norepinephrine system. Current biology : CB 25, R1051-R1056.

Semyanov, A., Henneberger, C., and Agarwal, A. (2020). Making sense of astrocytic calcium signals from acquisition to interpretation. Nature reviews Neuroscience 21, 551-564.

Serrano, A., Haddjeri, N., Lacaille, J.C., and Robitaille, R. (2006). GABAergic network activation of glial cells underlies hippocampal heterosynaptic depression. The Journal of neuroscience : the official journal of the Society for Neuroscience 26, 5370-5382.

Shen, W., Nikolic, L., Meunier, C., Pfrieger, F., and Audinat, E. (2017). An autocrine purinergic signaling controls astrocyte-induced neuronal excitation. Scientific reports 7, 11280.

Shigetomi, E., Bowser, D.N., Sofroniew, M.V., and Khakh, B.S. (2008). Two forms of astrocyte calcium excitability have distinct effects on NMDA receptor-mediated slow inward currents in pyramidal neurons. The Journal of neuroscience : the official journal of the Society for Neuroscience 28, 66596663.

Shigetomi, E., Jackson-Weaver, O., Huckstepp, R.T., O'Dell, T.J., and Khakh, B.S. (2013). TRPA1 channels are regulators of astrocyte basal calcium levels and long-term potentiation via constitutive $D$-serine release. The Journal of neuroscience : the official journal of the Society for Neuroscience 33, 1014310153.

Srinivasan, R., Huang, B.S., Venugopal, S., Johnston, A.D., Chai, H., Zeng, H., Golshani, P., and Khakh, B.S. (2015). Ca2+ signaling in astrocytes from Ip3r2-/- mice in brain slices and during startle responses in vivo. Nature Neuroscience 18, 708-717. 
1012 Takata, N., Mishima, T., Hisatsune, C., Nagai, T., Ebisui, E., Mikoshiba, K., and Hirase, H. (2011). Astrocyte calcium signaling transforms cholinergic modulation to cortical plasticity in vivo. The Journal of neuroscience : the official journal of the Society for Neuroscience 31, 18155-18165. Tello-Ramos, M.C., Branch, C.L., Kozlovsky, D.Y., Pitera, A.M., and Pravosudov, V.V. (2019). Spatial memory and cognitive flexibility trade-offs: to be or not to be flexible, that is the question. Animal Behaviour 147, 129-136. Traynelis, S.F., Wollmuth, L.P., McBain, C.J., Menniti, F.S., Vance, K.M., Ogden, K.K., Hansen, K.B., Yuan, H., Myers, S.J., and Dingledine, R. (2010). Glutamate receptor ion channels: structure, regulation, and function. Pharmacological reviews 62, 405-496. Wobrock, T., Ecker, U.K., Scherk, H., Schneider-Axmann, T., Falkai, P., and Gruber, O. (2009). Cognitive impairment of executive function as a core symptom of schizophrenia. The world journal of biological

1024

1025 psychiatry : the official journal of the World Federation of Societies of Biological Psychiatry 10, 442-451. Wolosker, H., Balu, D.T., and Coyle, J.T. (2017). Astroglial Versus Neuronal D-Serine: Check Your Controls! Trends in neurosciences. Woo, D.H., Han, K.S., Shim, J.W., Yoon, B.E., Kim, E., Bae, J.Y., Oh, S.J., Hwang, E.M., Marmorstein, A.D., Bae, Y.C., et al. (2012). TREK-1 and Best1 channels mediate fast and slow glutamate release in astrocytes upon GPCR activation. Cell 151, 25-40.

Yang, J., Vitery, M.D.C., Chen, J., Osei-Owusu, J., Chu, J., and Qiu, Z. (2019). Glutamate-Releasing SWELL1 Channel in Astrocytes Modulates Synaptic Transmission and Promotes Brain Damage in Stroke. Neuron 102, 813-827 e816.

1032 Zerbi, V., Floriou-Servou, A., Markicevic, M., Vermeiren, Y., Sturman, O., Privitera, M., von Ziegler, L., 1033 Ferrari, K.D., Weber, B., De Deyn, P.P., et al. (2019). Rapid Reconfiguration of the Functional

1034 Connectome after Chemogenetic Locus Coeruleus Activation. Neuron 103, 702-718 e705.

1035

1036

1037 Zhang, M., and Wang, H. (2013). Mice overexpressing type 1 adenylyl cyclase show enhanced spatial memory flexibility in the absence of intact synaptic long-term depression. Learning \& memory 20, 352357.

1038 Zhang, Z., Gong, N., Wang, W., Xu, L., and Xu, T.L. (2008). Bell-shaped D-serine actions on hippocampal long-term depression and spatial memory retrieval. Cerebral cortex 18, 2391-2401. 